\title{
Review Article \\ Experimental Results on Charge Fluctuations in Heavy-Ion Collisions
}

\author{
D. K. Mishra, ${ }^{1}$ P. Garg, ${ }^{2}$ P. K. Netrakanti, ${ }^{1}$ L. M. Pant, ${ }^{1}$ and A. K. Mohanty ${ }^{1}$ \\ ${ }^{1}$ Nuclear Physics Division, Bhabha Atomic Research Center, Mumbai 400085, India \\ ${ }^{2}$ Department of Physics and Astronomy, Stony Brook University, SUNY, Stony Brook, NY 11794-3800, USA \\ Correspondence should be addressed to D. K. Mishra; dkmishra@rcf.rhic.bnl.gov
}

Received 7 September 2016; Revised 29 November 2016; Accepted 30 November 2016; Published 17 January 2017

Academic Editor: Shi-Hai Dong

Copyright (C) 2017 D. K. Mishra et al. This is an open access article distributed under the Creative Commons Attribution License, which permits unrestricted use, distribution, and reproduction in any medium, provided the original work is properly cited. The publication of this article was funded by SCOAP $^{3}$.

\begin{abstract}
We present a subset of experimental results on charge fluctuation from the heavy-ion collisions to search for phase transition and location of critical point in the QCD phase diagram. Measurements from the heavy-ion experiments at the SPS and RHIC energies observe that total charge fluctuations increase from central to peripheral collisions. The net-charge fluctuations in terms of dynamical fluctuation measure $\nu_{(+-, \text {dyn })}$ are studied as a function of collision energy $\left(\sqrt{s_{N N}}\right)$ and centrality of the collisions. The product of $\nu_{(+-, \text {dyn })}$ and $\left\langle N_{\mathrm{ch}}\right\rangle$ shows a monotonic decrease with collision energies, which indicates that at LHC energy the fluctuations have their origin in the QGP phase. The fluctuations in terms of higher moments of net-proton, net-electric charge, and net-kaon have been measured for various $\sqrt{\boldsymbol{s}_{N N}}$. Deviations are observed in both $S \sigma$ and $\kappa \sigma^{2}$ for net-proton multiplicity distributions from the Skellam and hadron resonance gas model for $\sqrt{s_{N N}}<39 \mathrm{GeV}$. Higher moment results of the net-electric charge and net-kaon do not observe any significant nonmonotonic behavior as a function of collision energy. We also discuss the extraction of the freeze-out parameters using particle ratios and experimentally measured higher moments of net-charge fluctuations. The extracted freeze-out parameters from experimentally measured moments and lattice calculations are found to be in agreement with the results obtained from the fit of particle ratios to the thermal model calculations.
\end{abstract}

\section{Introduction}

The main goal of the high energy heavy-ion collisions is to study the phase structure of the quantum chromodynamic (QCD) phase diagram at finite temperature $(T)$ and baryon chemical potential $\left(\mu_{B}\right)[1-5]$. Several theoretical models suggest that the QCD phase diagram may contain a firstorder phase transition line between the hadron gas (HG) phase and Quark-Gluon phase which ends at the critical point towards high $T$ and lower $\mu_{B}$ [6-10]. Experimental programs have been performed at SPS and beam-energy scan (BES-I) program at RHIC to search for critical point and QGP-HG phase transition. In future, the upcoming program at RHIC (BES-II) [11], FAIR [12], NICA [13], and J-PARC [14] will also contribute to the physics at large $\mu_{B}$. The location of the QCD critical point can be explored by systematically varying $T$ and $\mu_{B}$. Experimentally, by changing the center of mass energy one can control $T$ and $\mu_{B}$ of the system and hence enables us to scan different sectors of the phase diagram.

One of the most striking signatures of such a QGPHG phase transition could be a strong modification in the fluctuations of specific observables measured on an eventby-event basis $[15,16]$. In principle, any observable that is not globally conserved fluctuates. Although most of these fluctuations are trivial and are of statistical origin, it is important to find out the dynamically relevant event-toevent fluctuation that enables the search for a possible critical point and a first-order coexistence region in the QCD phase diagram [17]. Over the past two decades quite a number of such observables have been suggested for clarifying the evolution of the system formed in heavy-ion collisions. These either refer to the signals from the plasma that are supposed to survive the phase transition or to the observables that experience strong fluctuation during the phase transition or 
close to the critical point. Most commonly measured eventby-event fluctuations in heavy-ion collision experiments are particle ratios $(K / \pi, p / \pi$, etc. $)$, transverse energy $\left(E_{T}\right)$, mean transverse momentum $\left\langle p_{T}\right\rangle$, and particle multiplicity $(N)$ fluctuations [18-21]. Predictions suggest that enhanced multiplicity fluctuations are connected to the production of QGP droplets, and suppression of fluctuation is connected to the nucleation process in a first-order QGP-HG phase transition. This may happen because of the rapid freeze-out just after the phase transition $[15,22]$. An isothermal compressibility of the system can be considered to understand the sensitivity of the measured particle multiplicity to the phase transition [23]. The isothermal compressibility is defined as $k_{T}=$ $-1 / V(\delta V / \delta P)_{T}$, where $P, V$, and $T$ are the pressure, volume, and temperature of the system, respectively. In order to relate the compressibility to the measurements of multiplicity fluctuations, we assume that relativistic heavy-ion collisions can be described as a thermal system in the Grand Canonical Ensemble (GCE) [24]. The GCE is the most appropriate description as only part of the particles from the system around mid-rapidity are measured by the experiments. The energy and conserved quantum numbers in this region can be exchanged with the rest of the system that serves as a heat bath [25]. Several other studies have applied Canonical and Microcanonical ensembles to the multiplicity fluctuations too [26-28]. In the GCE, the isothermal compressibility is directly related to the variance of the particle multiplicity as follows:

$$
\left\langle(N-\langle N\rangle)^{2}\right\rangle=\operatorname{var}(N)=\frac{k_{B} T\langle N\rangle^{2}}{V} k_{T},
$$

where $N$ is the particle multiplicity, $\langle N\rangle=\mu_{N}$ is the mean of the multiplicity distribution, and $k_{B}$ is the Boltzmann constant [29]. Here, multiplicity fluctuation measurements are presented in terms of the scaled variance, $\omega_{N}$, as [30]

$$
\omega_{N}=\frac{\operatorname{var}(N)}{\mu_{N}}=k_{B} T \frac{\mu_{N}}{V} k_{T} .
$$

In a continuous or second-order phase transition, the compressibility diverges at the critical point. Near the critical point, this divergence is described by a power law in the variable $\epsilon=\left(T-T_{c}\right) / T_{c}$, where $T_{c}$ is the critical temperature. Hence, the relationship between multiplicity fluctuations and the compressibility can be exploited to search for a signature of critical behavior by looking for the expected power law scaling of the compressibility:

$$
k_{T} \propto\left(\frac{T-T_{c}}{T_{c}}\right)^{-\gamma} \propto|\epsilon|^{-\gamma},
$$

where $\gamma$ is the critical exponent for isothermal compressibility [29]. Recent studies [31, 32] show the behavior of the quark number susceptibility, $\chi_{q}$, which is related to the value of the isothermal compressibility of the system. They predict that its value will increase by at least an order of magnitude close to the QCD critical point. As discussed above, the scaled variance is proportional to $k_{T}$; hence, the measurements of charged particle multiplicity are expected to be a sensitive probe for critical behavior.
If the system approaches close enough to the critical line for a long enough time period, then critical phenomena could be observed through the measurement of multiplicity fluctuations [8]. Subsequently, it may also be possible to determine the critical exponents of the system. Observation of critical behavior in heavy-ion collisions and the subsequent measurement of the critical exponents could determine the universality class in which QCD is grouped, providing essential constraints for the models [30].

The fluctuations of conserved quantities are predicted to be one of the most sensitive signals of the QGP formation and phase transition, which may provide complementary understanding of strong interactions, apart from other QGP signatures $[15,33]$. It has been argued that entropy conserving hadronization of plasma of quarks and gluons should produce a final state characterized by a dramatic reduction of the net-charge fluctuations in QGP phase as compared to that of a hadron gas. Further, prediction relies on the notion that quark-quark correlations can be neglected, and hadronization of gluons produces pairs of positive and negative particles not contributing to the net-charge fluctuations. It has also been suggested that the excitation function of conserved numbers like net-baryon, net-charge, and net-strangeness fluctuations should show a nonmonotonic behavior, as a possible signature of QCD critical end point (CEP) $[1,34,35]$. In the thermodynamic limit, the correlation length $(\xi)$ diverges at CEP [1]. The experimentally measured moments of the net-baryon, net-charge, and net-strangeness distributions are related to the higher power of $\xi$ of the system and hence these moments can be used to look for signals of a phase transition and critical point [36, 37]. Also, the comparison of experimentally measured cumulants with the lattice calculations enables us to extract the freeze-out parameters, that is, freeze-out temperature $\left(T_{f}\right)$ and $\mu_{B}$ of the system produced in heavy-ion collisions $[38,39]$. In recent years, lots of efforts have been put on both theoretical and experimental fronts to study the fluctuation of conserved quantities.

This review is organized as follows. In the following section, we discuss the total charge fluctuations from various experiments. In Section 3, the results on net-charge fluctuation are presented, which include dynamical fluctuation measure $v_{(+-, \text {dyn })}$, higher moments of net-proton, net-electric charge, and net-kaon fluctuations. Towards end of Section 3, extraction of freeze-out parameters using higher moments is discussed. Finally, in Section 4, we summarize our observations.

\section{Total Charge Fluctuation}

In a thermodynamical system of strongly interacting matter formed in the heavy-ion collisions, the fluctuations of particle multiplicities, mean transverse momentum $\left(\left\langle p_{T}\right\rangle\right)$, transverse energy $\left(E_{T}\right)$, and other global observables are related to the fundamental properties of the system, such as specific heat, chemical potential, and compressibility. These observables either refer to signals from the plasma that are supposed to survive the phase transition or to observables that experience strong fluctuations during the 
phase transition or close to the critical point. The existence of a critical point at the QCD phase transition has been associated with the large event-by-event fluctuations of above observables. As far as observables are concerned, electric charge fluctuations have been measured over a wide range of collision energies, from CERN SPS to RHIC and LHC energies. Enhanced fluctuations in neutral to charged pions have been predicted as a signature of the formation of Disoriented Chiral Condensates (DCC) [40, 41]. The relative fluctuation $\omega_{N}$ which can be extracted from experimental data has contributions from both statistical and dynamical sources. In order to extract the dynamical part associated with new physics from the observed fluctuations, one has to understand the contributions from statistical and other known sources. Some of the known sources of fluctuations contributing to the observed experimental value of scaled variance $\left(\omega_{N}\right)$ include finite particle multiplicity, effect of limited acceptance of the detectors, impact parameter fluctuations, fluctuations in the number of primary collisions, effects of rescattering of secondaries, resonance decays, and Bose-Einstein correlations [42].

The relative fluctuation $\omega_{\mathrm{ch}}$ is defined as

$$
\omega_{\mathrm{ch}}=\frac{\left\langle N_{\mathrm{ch}}^{2}\right\rangle-\left\langle N_{\mathrm{ch}}\right\rangle^{2}}{\left\langle N_{\mathrm{ch}}\right\rangle}=\frac{\operatorname{var}\left(N_{\mathrm{ch}}\right)}{\left\langle N_{\mathrm{ch}}\right\rangle}
$$

where $N_{\mathrm{ch}}$ is the charged particle multiplicity and $\omega_{\mathrm{ch}}$ is also known as scaled variance. If the multiplicity distribution is Poissonian, the scaled variance is 1.0. Figure 1 shows the comparison of the relative fluctuation $\omega_{\mathrm{ch}}$ of the charge particle multiplicity as a function of collision centrality which is related to number of participants $\left(N_{\text {part }}\right)$ in $\mathrm{Pb}+\mathrm{Pb}$ collisions at $158 \mathrm{AGeV}$ [40]. The error on $\omega_{\mathrm{ch}}$ calculated in the model is mainly due to the error on the mean number of charged particles in nucleon-nucleon interactions, the error in the number of participants calculated, and the uncertainty in the calculated transverse energy [40]. The experimental data are compared with the model calculations. It is observed that the relative fluctuations increase from central to peripheral collisions. The observed charge particles multiplicity fluctuations have been found to well agree with the results obtained from a simple participant model [45]. In the participant model, the particle multiplicity $N$ may be expressed as

$$
N=\sum_{i=1}^{N_{\text {part }}} n_{i},
$$

where $N_{\text {part }}$ is the number of participants in the collision and $n_{i}$ is the number of particles produced by the $i$ th participant within the detector acceptance. The mean value of $n_{i}$ is the ratio of the average multiplicity measured in the detector acceptance to the average number of participants, $\langle n\rangle=$ $\langle N\rangle /\left\langle N_{\text {part }}\right\rangle$. Hence, fluctuation in the particle multiplicity $N$ will have contributions due to fluctuations in $N_{\text {part }},\left(\omega_{N_{\text {part }}}\right)$ and also due to the fluctuations in the number of particles

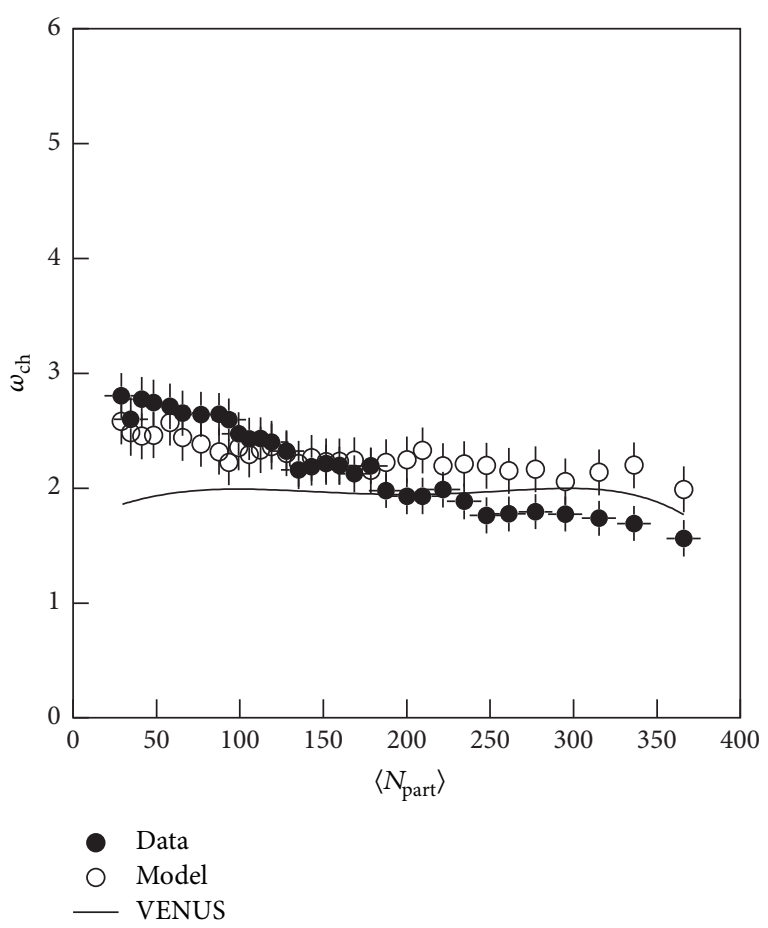

FIgURE 1: The relative fluctuations, $\omega_{\mathrm{ch}}$, of the event-by-event measured charged particle multiplicity as a function of number of participants $\left(N_{\text {part }}\right)$ in $\mathrm{Pb}+\mathrm{Pb}$ collisions at $158 \mathrm{AGeV}$ [40]. The combined (statistical and systematic) errors on $\omega_{\mathrm{ch}}$ from the experimental data are shown along with the data points. Details on error estimation are in [40]. The experimental data are compared to calculations from a participant model and those from VENUS event generator.

produced per participant $\left(\omega_{n}\right)$. The multiplicity fluctuation in the participant model can be expressed as [40]

$$
\omega_{N}=\omega_{n}+\langle n\rangle \omega_{N_{\text {part }}} .
$$

Another experiment at SPS also performed similar study shown in Figure 2 [43]. The scaled variance $(\operatorname{Var}(n) /\langle n\rangle)$, where $\operatorname{Var}(n)=\left(\left\langle n^{2}\right\rangle-\langle n\rangle^{2}\right)$ is the variance of the distribution and $n$ is the multiplicity of the particles. The scaled variance of positive, negative, and total charged particles as a function of centrality is shown in Figure 2. The experimental data is compared with the model calculations. The results from different models (HIJING [46], HSD [47], UrQMD [48], and VENUS [49]) are almost independent of centrality and behave like a Poisson expectation. However, the experimental data points indicate strong dependence on centrality. The scaled variance increases from central to peripheral collisions. The measured centrality dependence can be reproduced in superposition models with the assumption of contributions from target participants to the particle production in the forward hemisphere $[43,44]$. Figure 3 shows the centrality $\left(N_{\text {part }}\right)$ dependence of scaled variance at $\sqrt{s_{N N}}=62.4$ and $200 \mathrm{GeV}$ in $\mathrm{Au}+\mathrm{Au}$ collisions at RHIC [30]. The shaded regions represent the systematic uncertainties from the reference range. The statistical uncertainties are shown along with the data points. Here, $\omega_{\text {ch,dyn }}$ represents the estimate of the remaining 


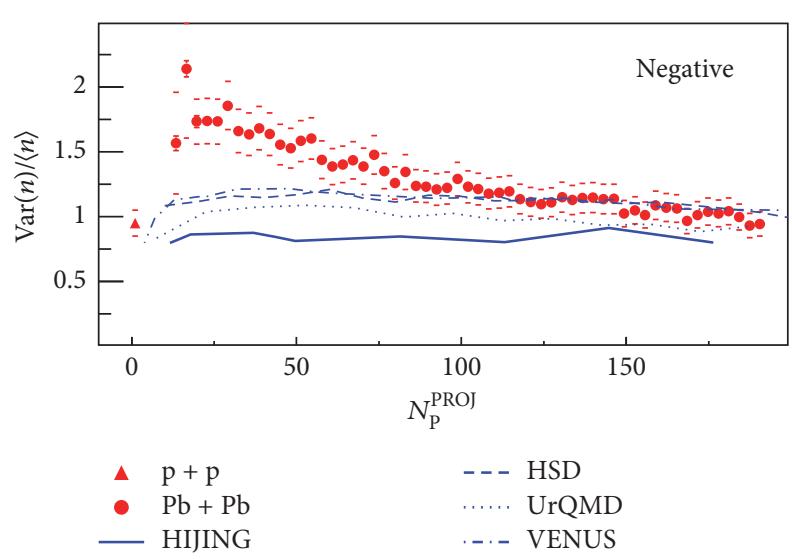

(a)

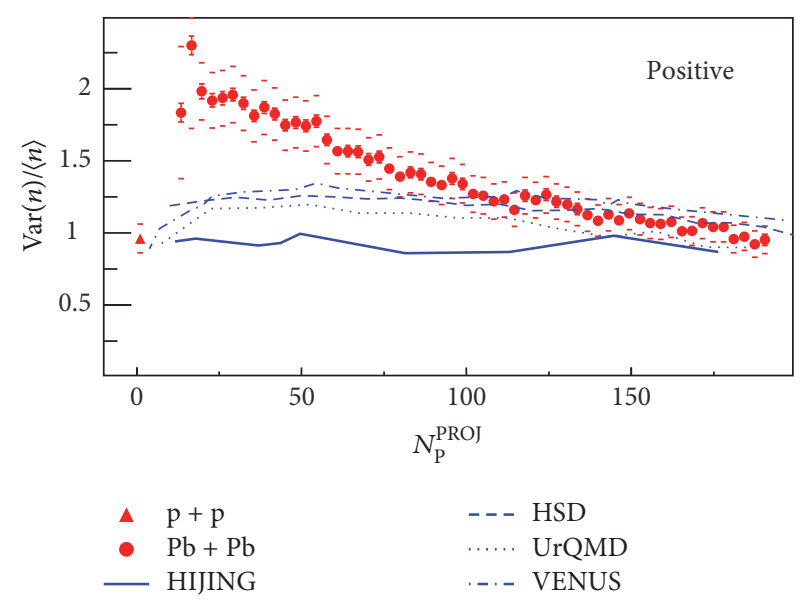

(b)

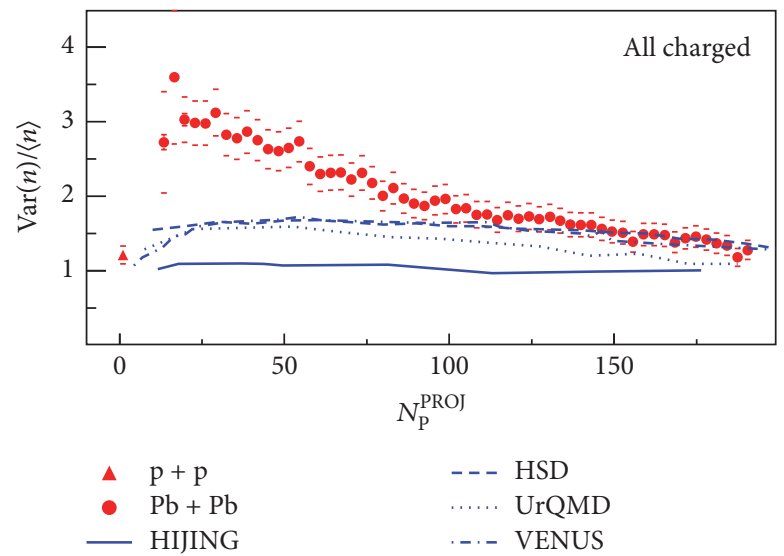

(c)

FIGURE 2: The scaled variance of the multiplicity distribution for negatively (a), positively (b), and total (c) charged particles as a function of centrality in terms of number of projectile participants in $\mathrm{Pb}+\mathrm{Pb}$ collisions at $158 \mathrm{AGeV}$ [43]. The experimental results are compared with model simulations in the NA49 acceptance (HSD and UrQMD predictions were taken from [44]). The statistical errors are smaller than the symbols (except for the most peripheral points). The horizontal bars indicate the systematic uncertainties.

dynamical multiplicity fluctuations. For all centralities, the scaled variance lies above the Poisson expectation of 1.0. At these energies, also the scaled variance increases from central to peripheral collisions. Hence, similar centrality dependence has been observed by the experiments at the SPS and RHIC energies. The absence of large dynamical fluctuation in excess of the participant superposition model indicates that there is no evidence of critical behavior related to the compressibility observable.

\section{Net-Charge Fluctuations}

One of the proposed signatures to search for the phase transition from hadronic to partonic medium is to study the netcharge fluctuations in heavy-ion collisions. The fluctuation in the net-charge depends on the square of the charges and hence strongly depend on which phase it originates from. The charge carriers in the QGP phase are quarks having fractional charges, while in hadronic phase the constituents have unit charge; hence, the measure of the fluctuations in the netcharge is expected to be different in these two cases [22]. In this section, we discuss the net-charge fluctuations using different fluctuation measures such as $v_{(+-, \text {dyn })}$ and higher moments.

3.1. Fluctuation Study Using $v_{(+-, d y n)}$. The net-charge fluctuations are expected to be smaller in the QGP phase as compared to the hadron gas (HG) phase [22]. The net-charge fluctuations may get affected by uncertainties due to volume fluctuation, exact local charge conservation, or repulsive forces among hadrons [39]. However, it is important to know whether these fluctuations may or may not survive the evolution of the system in the heavy-ion collisions. The collision volume is not directly measured in the experiment which may lead to additional geometrical fluctuations. One can get rid of volume fluctuation by considering the ratios of the number of positive $\left(N_{+}\right)$to negative $\left(N_{-}\right)$particles, $R=N_{+} / N_{-}$. The ratio fluctuation is defined by $\left\langle\delta R^{2}\right\rangle=\left\langle R^{2}\right\rangle-\langle R\rangle^{2}$. 


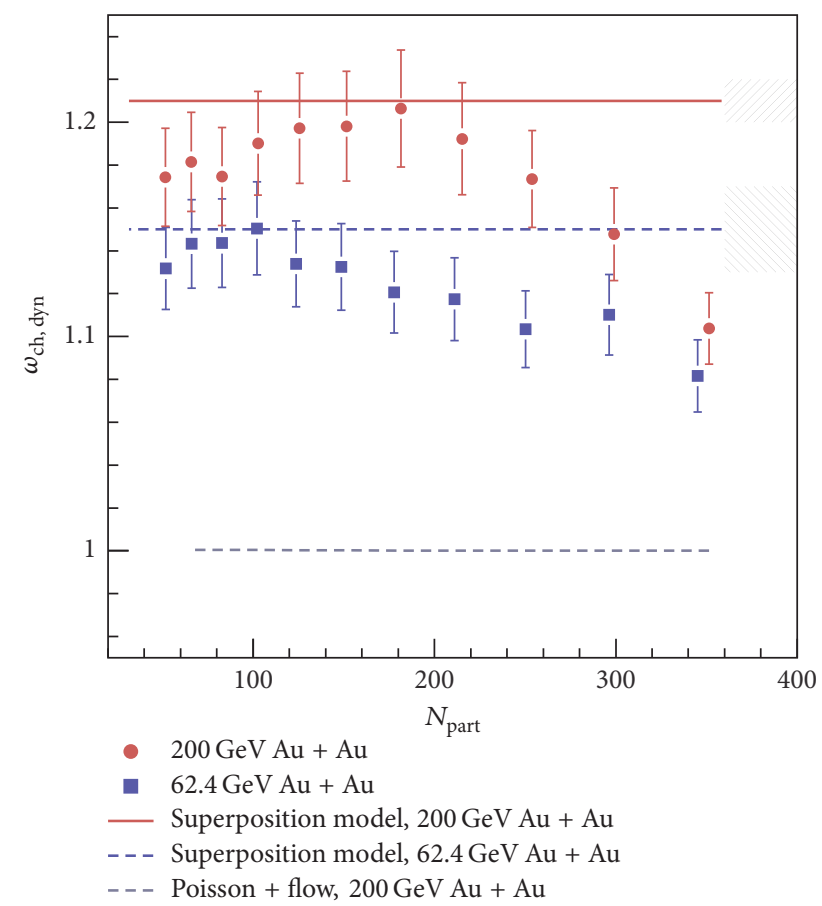

FIgURE 3: Centrality dependence of the scaled variance in $\mathrm{Au}+\mathrm{Au}$ collisions for $0.2<p_{T}<2.0 \mathrm{GeV} / c$ for $\sqrt{s_{N N}}=62.4$ and $200 \mathrm{GeV}$ [30]. Results from the superposition model are overladed with the shaded regions representing a one-standard deviation range of the prediction for the fluctuation magnitude derived from $\mathrm{p}+\mathrm{p}$ collision data. Contributions from noncorrelated particle emission with the Poisson distribution of the scaled variance with the addition of elliptic flow in $200 \mathrm{GeV} \mathrm{Au}+\mathrm{Au}$ collisions are also shown.

Since the fluctuation of the number of charged particles is a measure of the entropy of the system, another fluctuation observable $D$-measure of the net-charge provides a measure of charge fluctuations per unit entropy and is related to the ratio $R$ as

$$
\begin{aligned}
D & =\left\langle N_{\mathrm{ch}}\right\rangle\left\langle\delta R^{2}\right\rangle \\
& =\frac{4}{\left\langle N_{\mathrm{ch}}\right\rangle}\left\langle\delta N_{+}^{2}+\delta N_{-}^{2}-2 \delta N_{+} \delta N_{-}\right\rangle \approx \frac{4\left\langle\delta Q^{2}\right\rangle}{\left\langle N_{\mathrm{ch}}\right\rangle},
\end{aligned}
$$

where $\left\langle\delta Q^{2}\right\rangle$ is the variance of the net-charge with $Q=N_{+}-$ $N_{-}$being the difference between +ve and -ve particles and $\left\langle N_{\mathrm{ch}}\right\rangle=\left\langle N_{+}+N_{-}\right\rangle$is the average number of charged particles measured within the experimental acceptance. Assuming the quark-quark interactions to be negligible, $D$ was found to be approximately 4 times smaller in QGP phase as compared to HG phase. It has been shown that $D=4$ for uncorrelated pion gas and reduces to 3 after taking the resonance decay into account [25]. For a QGP phase, $D$ is estimated between 1.0 and 1.5. Hence, $D$ can be used as a probe to distinguish between the QGP and HG phases. Unfortunately, the quantity $\left\langle\delta Q^{2}\right\rangle /\left\langle N_{\mathrm{ch}}\right\rangle$ depends on the experimental efficiency. In the experiments, the net-charge fluctuations are studied in terms of dynamical fluctuation measure $\nu_{(+-, \text {dyn })}$, which is found to be independent of detection efficiency. The quantity

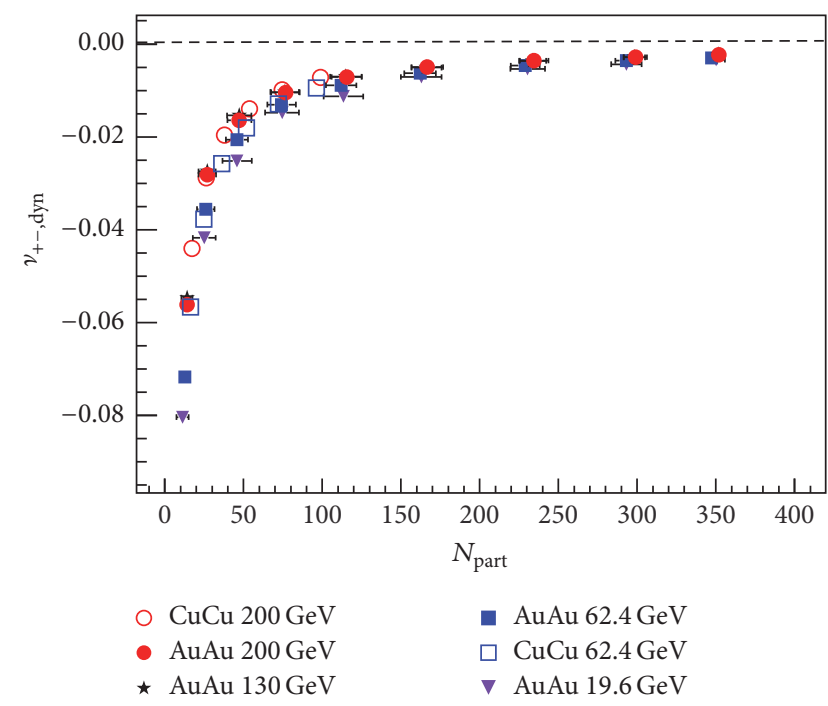

FIgure 4: Centrality $\left(N_{\text {part }}\right)$ and collision energy dependence of dynamical net-charge fluctuations, $v_{(+- \text {dyn })}$, of particles produced within pseudorapidity $|\eta|<0.5$ in $\mathrm{Cu}+\mathrm{Cu}$ and $\mathrm{Au}+\mathrm{Au}$ collisions at RHIC [51]. The combined (statistical and systematic) uncertainties are within the symbol size.

$\nu_{(+-, \text {dyn })}$ is a measure of the relative correlation strength of ,++-- , and +- particle pairs. A positive value of $\nu_{(+- \text {,dyn })}$ signifies the correlation of same charge pair, where a negative value indicates the dominant contribution from correlations between opposite charges. $\nu_{(+-, \text {dyn })}$ is defined by

$$
\begin{aligned}
\nu_{(+-, \text {dyn })}= & \frac{\left\langle N_{+}\left(N_{+}-1\right)\right\rangle}{\left\langle N_{+}\right\rangle^{2}}+\frac{\left\langle N_{-}\left(N_{-}-1\right)\right\rangle}{\left\langle N_{-}\right\rangle^{2}} \\
& -2 \frac{\left\langle N_{-} N_{+}\right\rangle}{\left\langle N_{-}\right\rangle\left\langle N_{+}\right\rangle},
\end{aligned}
$$

where $\left\langle N_{+}\right\rangle$and $\left\langle N_{-}\right\rangle$are the average number of positively and negatively charged particles within the detector acceptance. The $D$-measure and $\nu_{(+- \text {,dyn })}$ are related as $\left\langle N_{\text {ch }}\right\rangle \nu_{(+-, \text {dyn })} \approx D-4[50]$.

Figure 4 shows the centrality $\left(N_{\text {part }}\right)$ dependence of dynamical net-charge fluctuations $\left(\nu_{(+-, \text {dyn })}\right)$ in $\mathrm{Au}+\mathrm{Au}$ and $\mathrm{Cu}+\mathrm{Cu}$ collisions at different $\sqrt{s_{N N}} \cdot \nu_{(+-, \text {dyn })}$ values exhibit a monotonic dependence on $N_{\text {part }}$ and have small dependence on collision energy. For all the studied energies, the values of $\nu_{(+-, \text {dyn })}$ are negative, which indicates the dominance of correlation of positive and negative charged particle term in (8). The observed monotonic reduction of the magnitude of $\nu_{(+-, \text {dyn })}$ with increasing number of participants arises mainly due to the progressive dilution of two-particle correlation when the number of particle sources is increased.

In view of prediction for critical point in the QCD phase diagram in the range $10 \leq \sqrt{s_{N N}} \leq 60 \mathrm{GeV}[8,51]$, it can be argued that the reduction of fluctuation might be larger at lower beam energies. On the other hand, one may also argue that the volume of the QGP formed in $\mathrm{Au}+\mathrm{Au}$ collisions might increase at higher beam energies leading to reduced fluctuations. Hence, it is important to 


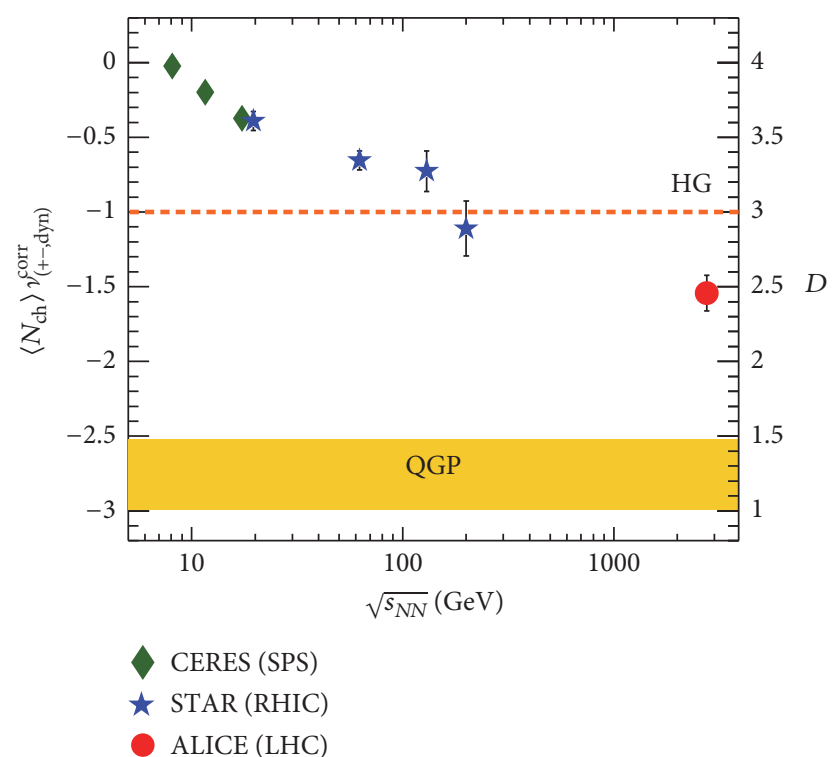

Figure 5: Energy dependence of net-charge fluctuations about midrapidity in central heavy-ion collisions at SPS [52], RHIC [51], and LHC [53] energies. Also shown are the expectations from a hadron resonance gas model and for a simple QGP picture [22]. The combined (statistical and systematic) errors are plotted along with the data points.

understand whether the fluctuations may be found to vary with beam energy thereby indicating the production of QGP above a critical threshold or with progressively increasing probability at higher energies. Figure 5 shows the product of $\nu_{(+-, \text {dyn })}$ and $\left\langle N_{\mathrm{ch}}\right\rangle$ (average number of charged particles) as a function of collision energies for $0-5 \%$ central collisions using the combined data from SPS, RHIC, and LHC energies [51-53]. Also, the collision energy dependence of $D$-measure is shown in the same Figure 5. It is observed that the fluctuation observable shows monotonic decrease in magnitude with increasing $\sqrt{s_{N N}}$ and approaches expectation for a simple QGP-like scenario [22] as we move from RHIC to LHC energies. It has been argued that measurements in lower $\sqrt{s_{N N}}$ (below $10 \mathrm{GeV}$ ) are dominated by baryons while at higher energies the meson and resonance production becomes increasingly dominant. This suggests that the change in dynamical net-charge fluctuations below $\sqrt{s_{N N}}=$ 19.6 GeV might be partially due to this shift in particle production dominance $[1,8,51,54-56]$. It is also argued that the differences between the fluctuation values below and above $19.6 \mathrm{GeV}$ may result from changes in the collision dynamics and final state interaction effects [8, 54-57]. For the highest RHIC energy, the measured value of fluctuation observable is close to the HG prediction, whereas at lower energies the results are higher than $\mathrm{HG}$ value. This may be due to the fact that at highest RHIC energy $\left(\sqrt{s_{N N}}=200 \mathrm{GeV}\right)$ the fluctuation may not be strong enough to be measured or because of the dilution of fluctuation during the evolution process. At LHC energy $\sqrt{s_{N N}}=2.76 \mathrm{TeV}$, the fluctuation observable value is significantly lower as compared to lower energies results. The fluctuations at the LHC energy might also have been diluted because of various effects; still these fluctuations are smaller than the theoretical expectations. In [22], it is shown that the $D$-measure value for hadron gas with resonance decay is $\simeq 3$ and for QGP phase is $\simeq 1.0-1.5$. The measured $D$-measure value is $2.3 \pm 0.21$ for $\Delta \eta=1.6$ at $\sqrt{s_{N N}}=2.76 \mathrm{TeV}$. There is a clear decreasing tendency of the $D$-measure value in the HG phase and approaches toward QGP expectation. This may indicate that the fluctuations have their origin in the QGP phase [53].

Several other observables already indicate that a hot and dense medium of color charges has been formed at RHIC and LHC energies. The net-charge fluctuation results suggest that either the observable $v_{(+- \text {,dyn })}$ is not sensitive enough to QGP physics or the process of hadronization washed out the QGP signal for this observable. It may be also noted that the theoretical results do not incorporate the acceptance effects and dynamical evolution of the system like, for example, the dilution of the signals in the hadronization process [58].

3.2. Fluctuation Study Using Higher Moments. In recent years, the beam-energy scan (BES) programs at SPS and RHIC have drawn much attention to map the quantum chromodynamics (QCD) phase diagram in terms of temperature $(T)$ and baryon chemical potential $\left(\mu_{B}\right)$. The location of the critical point can be explored by systematically varying $T$ and $\mu_{B}$, which can be experimentally achieved by varying the $\sqrt{s_{N N}}$ of the colliding ions. Several theoretical models suggest that excitation function of conserved numbers such as netbaryon, net-charge, and net-strangeness fluctuations should show a nonmonotonic behavior as a possible signature of QCD critical end point (CEP) [6-8]. In the vicinity of the QCD critical point, these variances are proportional to the square of the correlation length $(\xi)$, which is expected to diverge at the critical point $[34,59]$. However, the magnitude of the correlation length is limited by the system size and by finite time effects (critical slowing down), which could be as small as 2 to $3 \mathrm{fm}$. Hence, the contribution to the fluctuations from the critical point might be too weak as to be discovered experimentally, if only the second moments are measured. Therefore, it has been proposed to measure higher moments of the fluctuations which are expected to be much more sensitive to the critical point $[35,60,61]$. The moments of the net-baryon, net-charge, and net-strangeness distributions are related to $\xi$ of the system and hence these moments can be used to look for signals of a phase transition and critical point $[1,36,37]$. The variance $\left(\sigma^{2}\right)$ of these distributions is related to $\xi$ as $\sigma^{2} \sim \xi^{2}$, the skewness $(S)$ goes as $\xi^{4.5}$, and the kurtosis $(\kappa)$ is related as $\xi^{7}$. Hence, it is proposed to study the higher moments of conserved number distribution due to their stronger dependence on $\xi$.

Experimentally, the net-baryon number fluctuations are not directly measured, as all neutral baryons are not detected by most of the experiments. Hence, net-baryon fluctuations are accessible via measuring the net-proton distributions [62, 63]. The net-charge fluctuations are accessible by measuring the stable charged particles such as pions, kaons, and protons along with their antiparticles $[64,65]$. Similarly, the measurement of net-kaon fluctuations acts as a proxy for 


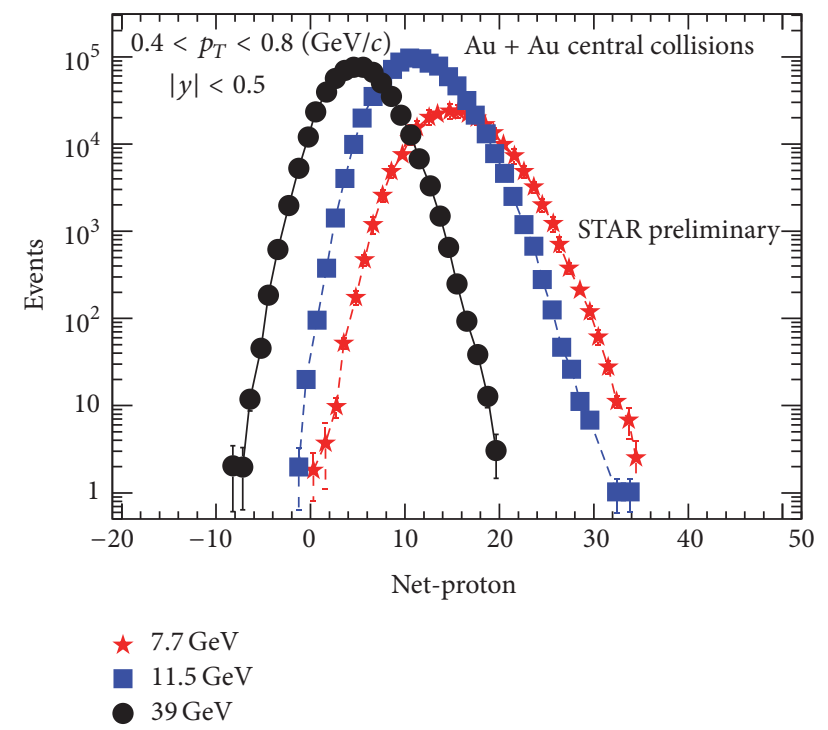

FIGURE 6: Uncorrected $\Delta N_{p}$ multiplicity distributions measured on an event-by-event basis in $\mathrm{Au}+\mathrm{Au}$ collisions at various $\sqrt{s_{N N}}$ for $0-5 \%$ collision centrality measured by STAR experiment.

net-strangeness fluctuations, because higher mass strange particles are not directly measured $[66,67]$. In the following subsections we discuss each of the conserved number fluctuations separately.

The moments of the event-by-event experimentally measured net distributions are related to the different order of the cumulants of the distribution as mean $(M)=C_{1} ; \sigma^{2}=$ $C_{2}=\left\langle(\Delta N)^{2}\right\rangle ; S=C_{3} / C_{2}^{3 / 2}=\left\langle(\Delta N)^{3}\right\rangle / \sigma^{3}$, and $\kappa=C_{4} / C_{2}^{2}=$ $\left\langle(\Delta N)^{4}\right\rangle / \sigma^{4}-3$, where $N$ is the multiplicity of the distribution and $\Delta N=N-M$. Hence, the ratios of the cumulants are related to the moments as $\sigma^{2} / M=C_{2} / C_{1}, S \sigma=C_{3} / C_{2}, \kappa \sigma^{2}=$ $C_{4} / C_{2}$, and $S \sigma^{3} / M=C_{3} / C_{1}$. Further, the ratios of moments can be related to the susceptibilities of $n$th order $\left(\chi^{n}\right)$ obtained from the lattice QCD or from the HRG model calculations as $\sigma^{2} / M \sim \chi^{(2)} / \chi^{(1)}, S \sigma \sim \chi^{(3)} / \chi^{(2)}, \kappa \sigma^{2} \sim \chi^{(4)} / \chi^{(2)}$, and $S \sigma^{3} / M \sim \chi^{(3)} / \chi^{(1)}$ [68]. One advantage of measuring the ratios is that the volume dependence (which is not directly measured by the experiment) on the experimentally measured individual cumulants cancels out to first order in the ratios. Hence, experimentally measured quantities can be directly compared with the theoretical calculations $[68,69]$.

3.2.1. Net-Proton Fluctuation. Theoretical calculations have shown that net-proton fluctuations reflect the singularity of the charge and baryon number susceptibility as expected at the critical point [61]. Figure 6 shows the typical uncorrected net-proton $\left(\Delta N_{p}\right)$ distributions in $\mathrm{Au}+\mathrm{Au}$ collisions for 0 $5 \%$ centrality measured on event-by-event basis. The protons and antiprotons are measured within $p_{T}=0.4$ to $0.8 \mathrm{GeV} / c$ and $|y|<0.5$. At lower energies, the net-proton distributions are dominated by the contributions from the proton distributions. The mean value of the distribution increases with decrease in collision energy. Different moments which describe the shape of the distribution are extracted from the $\Delta N_{p}$ distribution.

Figure 7 shows the collision energy dependence of $S \sigma$ and $\kappa \sigma^{2}$ of net-proton distribution for $0-5 \%$ and $70-80 \%$ centralities in $\mathrm{Au}+\mathrm{Au}$ collisions. The statistical uncertainties are calculated using Delta theorem approach [70]. In the hot and dense medium, the baryon chemical potential $\mu_{B}$ decreases with increasing collision energies; hence, Figure 7 can be interpreted as $\mu_{B}$ dependence of the moments over the large range of $\mu_{B}(20-450 \mathrm{MeV})$ [63]. Deviations are observed for both $S \sigma$ and $\kappa \sigma^{2}$ from the Skellam and hadron resonance gas model for $\sqrt{s_{N N}}<39 \mathrm{GeV}$. Maximum deviations from Skellam expectation are observed for $\sqrt{s_{N N}}=19.6$ and $27 \mathrm{GeV}$. The experimental results are reasonably described by assuming independent production (IP) of $p$ and $\bar{p}$ indicating there is no apparent correlations between the proton and antiprotons for presented observables [63]. Therefore, one may ask, in spite of significantly correlated production due to baryon number, electric charge conservation, and kinematical correlations of proton and antiprotons, why do the measured cumulants follow the independent production model. This has been studied by introducing the correlation between two independently produced distributions. It is observed that experimentally measured cumulants will follow the IP model calculations even if the correlation coefficient is less than $20 \%$ [71]. However, $C_{4} / C_{2}\left(=\kappa \sigma^{2}\right)$ and $C_{3} / C_{1}\left(=S \sigma^{3} / M\right)$ values will follow the IP model for all the correlation coefficient values. The observation that the experimental data can be explained by the independent production of particles does not rule out the existence of the critical endpoint.

3.2.2. Net-Charge Fluctuation. The net-electric charge fluctuations are accessible through measuring fluctuations of stable charged particles $(\pi, K$, and $p)$. Net-electric charge fluctuations are more straightforward to measure experimentally than net-baryon number fluctuations. As discussed before, experimentally, net-baryon number fluctuations are accessible only through net-proton number fluctuations, but in lattice QCD calculations net-baryon fluctuations are calculated instead of net-proton fluctuations. While net-charge fluctuations are not as sensitive as net-baryon fluctuations to the theoretical parameters, both measurements are necessary for a full understanding of the theory [65]. Several studies suggest that the net-charge multiplicity distributions are better suited to extract the freeze-out parameters $\left(\mu_{B}\right.$ and $\left.T_{f}\right)$ and the location of the CEP as they directly probe a conserved quantum number (electric charge) $[37,73,74]$.

Figure 8 shows the collision energy dependence of efficiency corrected $S \sigma$ and $\kappa \sigma^{2}$ of the net-charge distributions for central $0-5 \% \mathrm{Au}+\mathrm{Au}$ collisions at RHIC. $S \sigma$ and $\kappa \sigma^{2}$ measurements from the PHENIX experiment are shown in (a) [65] and measurements from the STAR experiment are shown in (b) [64]. The statistical errors are calculated using Delta theorem method [70]. Here, we would like to briefly discuss about the statistical error calculations in both the STAR and PHENIX experiments. The errors on $S \sigma$ and $\kappa \sigma^{2}$ are correlated and are dependent on both variance $(\sigma)$ of the distribution and the number of events. As mentioned 


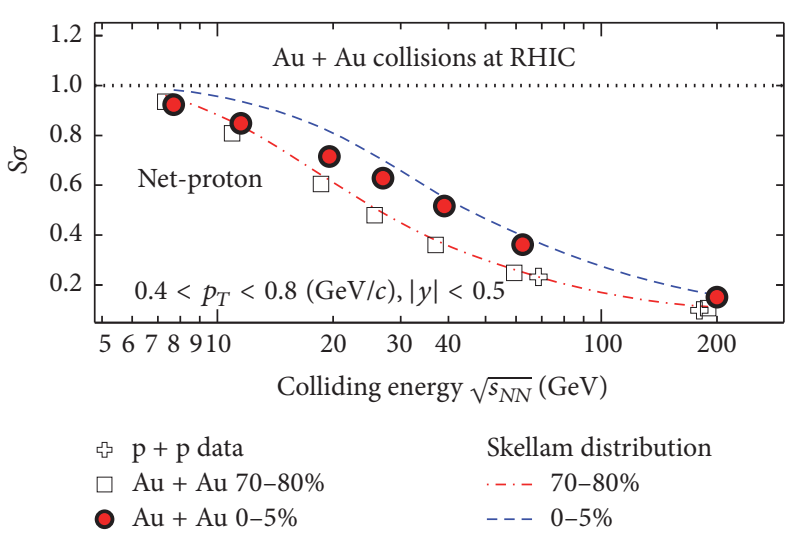

(a)

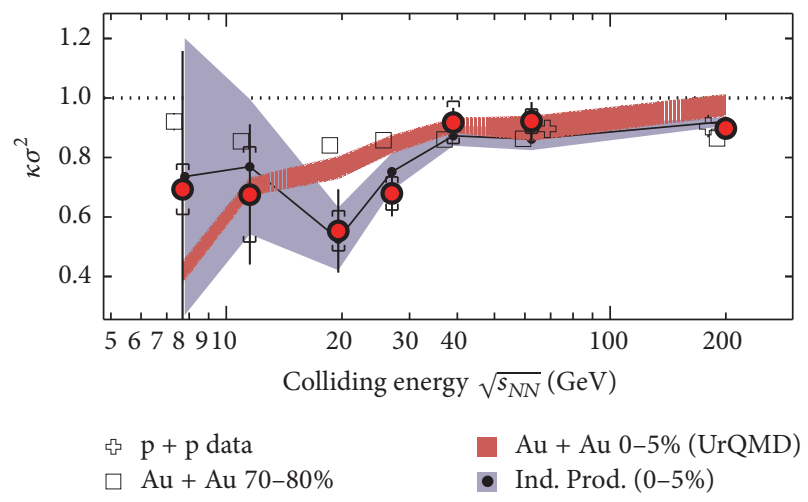

(b)

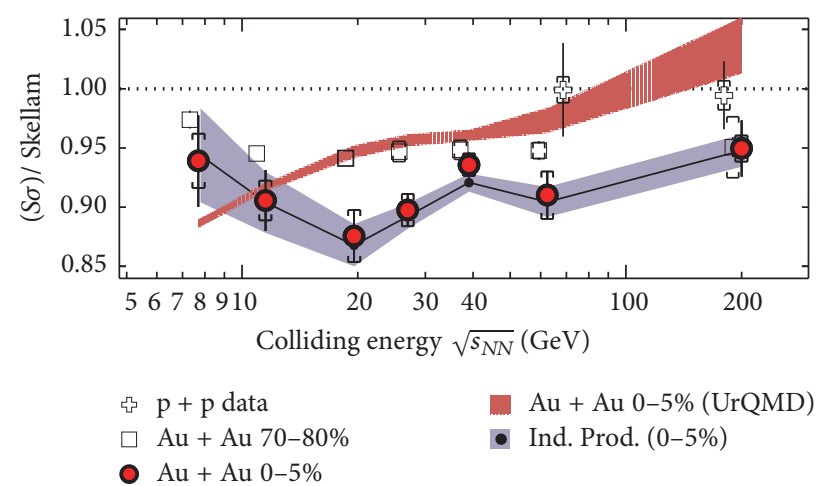

(c)

FIGURE 7: Collision energy and centrality dependence of efficiency corrected $S \sigma$ and $\kappa \sigma^{2}$ of net-proton distributions from Au $+\mathrm{Au}$ and $\mathrm{p}+\mathrm{p}$ collisions at RHIC [63]. Skellam distributions for corresponding collision centralities are shown for $S \sigma$. Shaded hatched bands are the results from UrQMD. In (b) and (c), the shaded solid bands are the expectations assuming independent proton and antiproton production. The HRG values for $\kappa \sigma^{2}$ and $S \sigma /$ Skellam are unity $[68,72]$. The error bars are statistical and caps are systematic errors.

in [70], the statistical errors are more dependent on $\sigma$ than the number of events. Hence, experiments having larger multiplicity distribution will have larger errors on $S \sigma$ and $\kappa \sigma^{2}$. Since STAR experiment has larger acceptance compared to PHENIX, it contributes to larger statistical error. Further, STAR net-charge distributions have larger $\sigma$ compared to net-proton distributions, which contributes to the larger statistical error in the net-charge results than the net-proton results, although the number of analyzed events are similar. $S \sigma$ values from both the experiments decrease with increasing $\sqrt{s_{N N}} \cdot \kappa \sigma^{2}$ values from PHENIX ((a), bottom panel) remain constant and positive, between $1.0<\kappa \sigma^{2}<2.0$ at all the collision energies within the statistical and systematic uncertainties. However, there is $25 \%$ increase of $\kappa \sigma^{2}$ values at lower energies below $\sqrt{s_{N N}}=39 \mathrm{GeV}$ compared to higher energies. Further, the $\kappa \sigma^{2}$ values from the STAR experiment are constant at all energies within uncertainties, except for $\sqrt{s_{N N}}=7.7 \mathrm{GeV}$ which shows a negative $\kappa \sigma^{2}$ value. The STAR experiment reported higher weighted mean $(2.4 \pm 1.2)$ of $\kappa \sigma^{2}$ values as compared to PHENIX $\kappa \sigma^{2}$ values. It is to be noted that results from the PHENIX experiment are measured within $0.3 \leq p_{T}(\mathrm{GeV} / c) \leq 2.0$ and $|\eta| \leq 0.35$ with $2 \times \pi / 2$ in azimuth, whereas results from the STAR experiment are within $0.2 \leq p_{T}(\mathrm{GeV} / c) \leq 2.0$ and $|\eta| \leq 0.5$ with full azimuth $(2 \pi)$. Different lower $p_{T}$ cut may be responsible for about $30 \%$ of the difference between two data sets [75]. More discussion on acceptance effect on higher moments can be found in $[72,75,76]$. However, net-charge results from both the experiments do not observe any significant nonmonotonic behavior in the products of moments as a function of collision energy.

3.2.3. Net-Kaon Fluctuation. Experimentally, the netstrangeness fluctuations are accessible through measuring the net-kaon fluctuations. Figure 9 shows the preliminary results on collision energy dependence of efficiency corrected $S \sigma$ and $\kappa \sigma^{2}$ of the net-kaon distributions for $0-5 \%$ and $70-$ $80 \%$ centralities in $\mathrm{Au}+\mathrm{Au}$ collisions measured by STAR experiment at RHIC [67]. With large uncertainties in the measurement, no significant deviation of the product of higher moments for net-kaon distributions as compared to the Poisson expectation has been observed at the measured energies. However, the upcoming RHIC BES-II with the upgraded detector system will help to reduce the uncertainties on the measurements and may find the location of the critical point. 

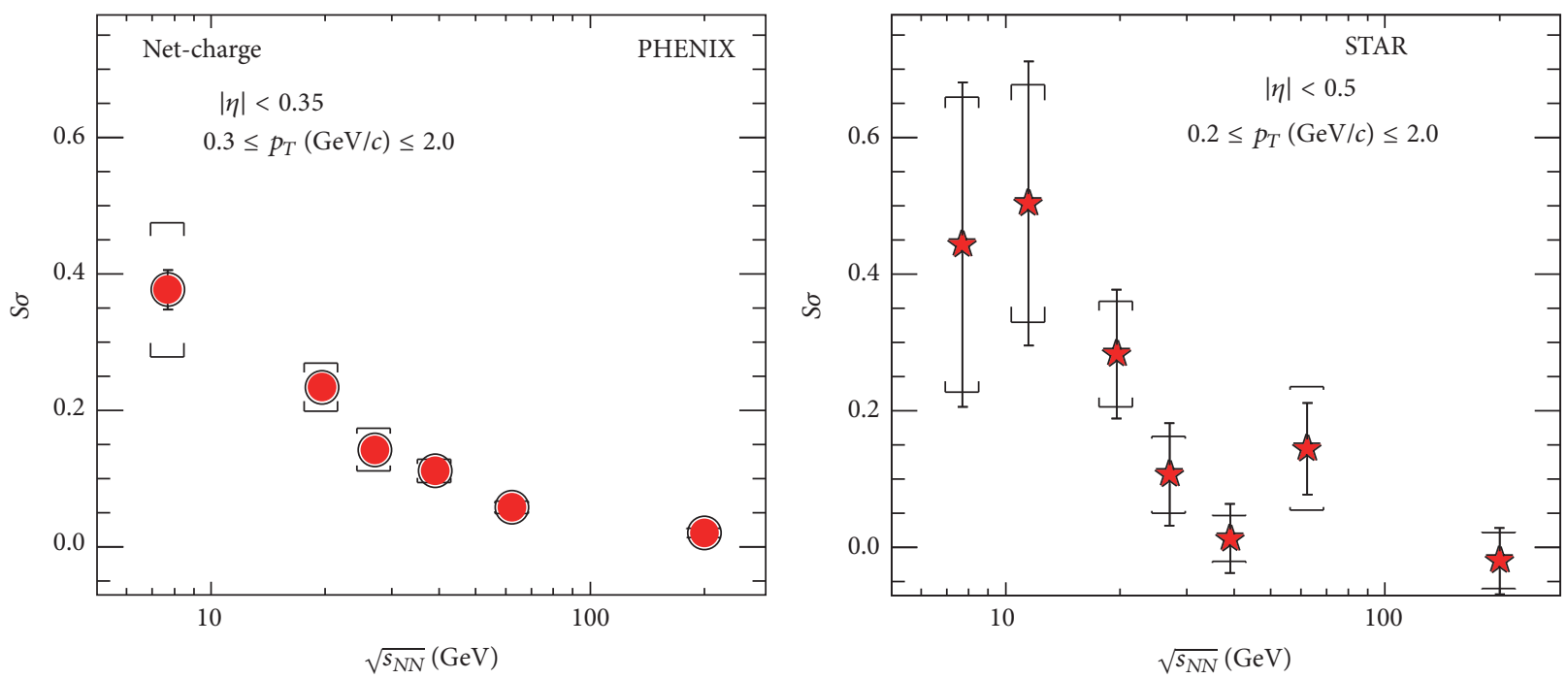

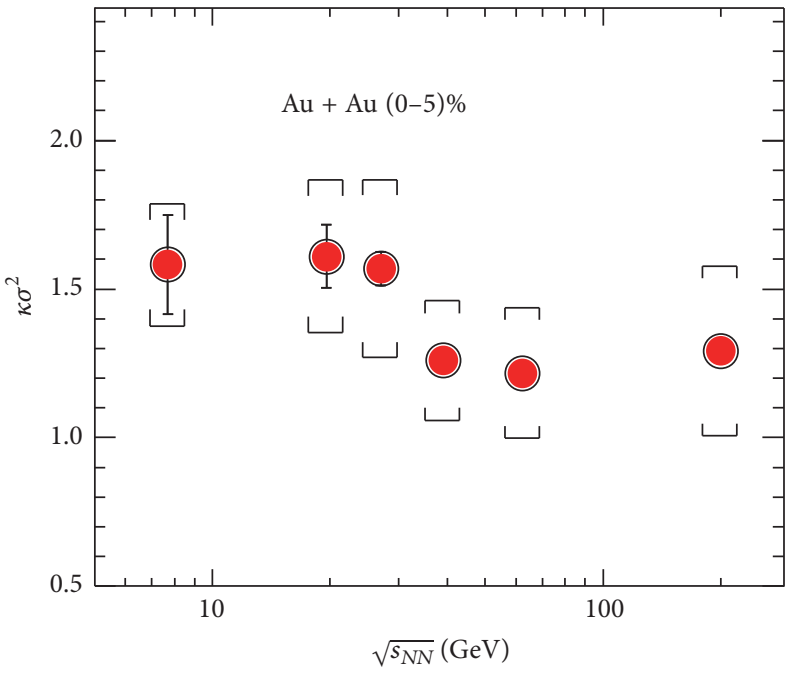

(a)

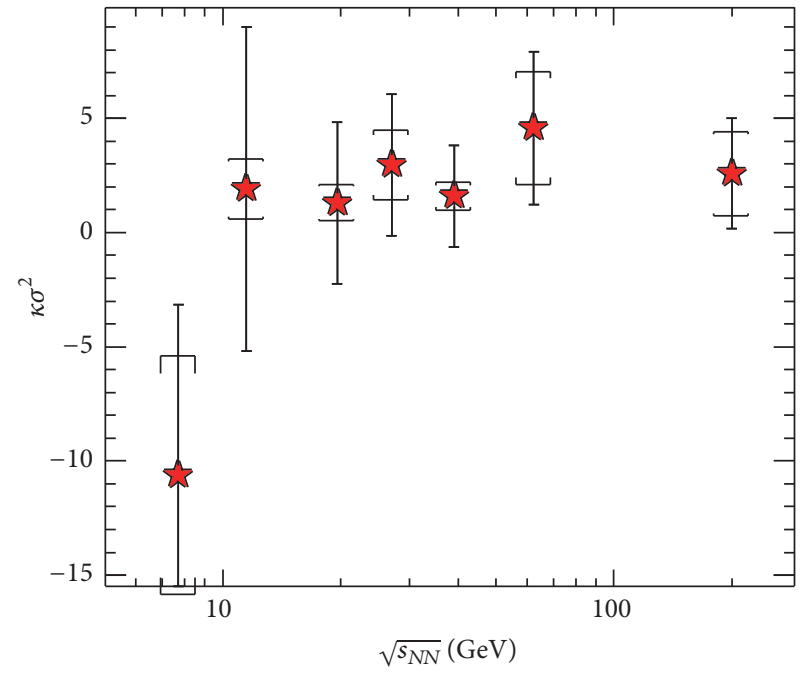

(b)

FIGURE 8: The energy dependence of efficiency corrected $S \sigma$ and $\kappa \sigma^{2}$ of net-charge distributions for central $(0-5 \%)$ Au + Au collisions at RHIC $[64,65]$. The error bars are statistical and caps are systematic uncertainties.

3.3. Extraction of Freeze-Out Parameters Using Higher Moments. Product of higher moments can be used to extract the freeze-out parameters $\left(\mu_{B}\right.$ and $\left.T_{f}\right)$ of the QCD phase diagram [37-39]. From the lattice calculations, it has been observed that the ratio of 1st to 2 nd cumulants $\left(=M / \sigma^{2}\right)$ shows a strong dependence on $\mu_{B}$ but varies little with $T$. On the other hand, the ratio of 3 rd to 1st cumulants $\left(=S \sigma^{3} / M\right)$ shows strong dependence on $T$ and has little dependence on $\mu_{B}[37,38]$. Hence, lattice calculations in combination with experimentally measured $S \sigma^{3} / M$ values at different collision energies can be used to extract $T_{f}$, whereas the measured $M / \sigma^{2}$ can be used to extract $\mu_{B}$. The collision energy dependence of freeze-out parameters $T_{f}$ and $\mu_{B}$ is shown in Figure 10. Freeze-out parameters extracted from different methods such as using particle ratios [77] and using cumulants with lattice [65] agree very well. Further, extracted
$T_{f}$ and $\mu_{B}$ values using lattice calculations and experimental data [65] are in agreement with the thermal model parameterization [77]. The freeze-out parameters extracted using combination of experimental data and HRG model [39] are also shown in Figure 10. The HRG calculations are performed in the same acceptance as the experiment. The extracted $\mu_{B}$ values using HRG and experimental measured cumulants are in agreement with other measurements, but the extracted $T_{f}$ are about 7-10 MeV lower as compared to the values extracted using lattice calculations $[39,65] . \mu_{B}$ values are consistent across different extraction procedure but there is some discrepancy in the value of $T_{f}$. One of the reasons may be that $\mu_{B}$ are extracted using ratio of 1st and 2 nd cumulants which are measured more precisely compared to higher cumulants. In [39], $\mu_{B}$ and $T_{f}$ are extracted using HRG for each $\sqrt{s_{N N}}$ by simultaneously reproducing the experimentally measured 

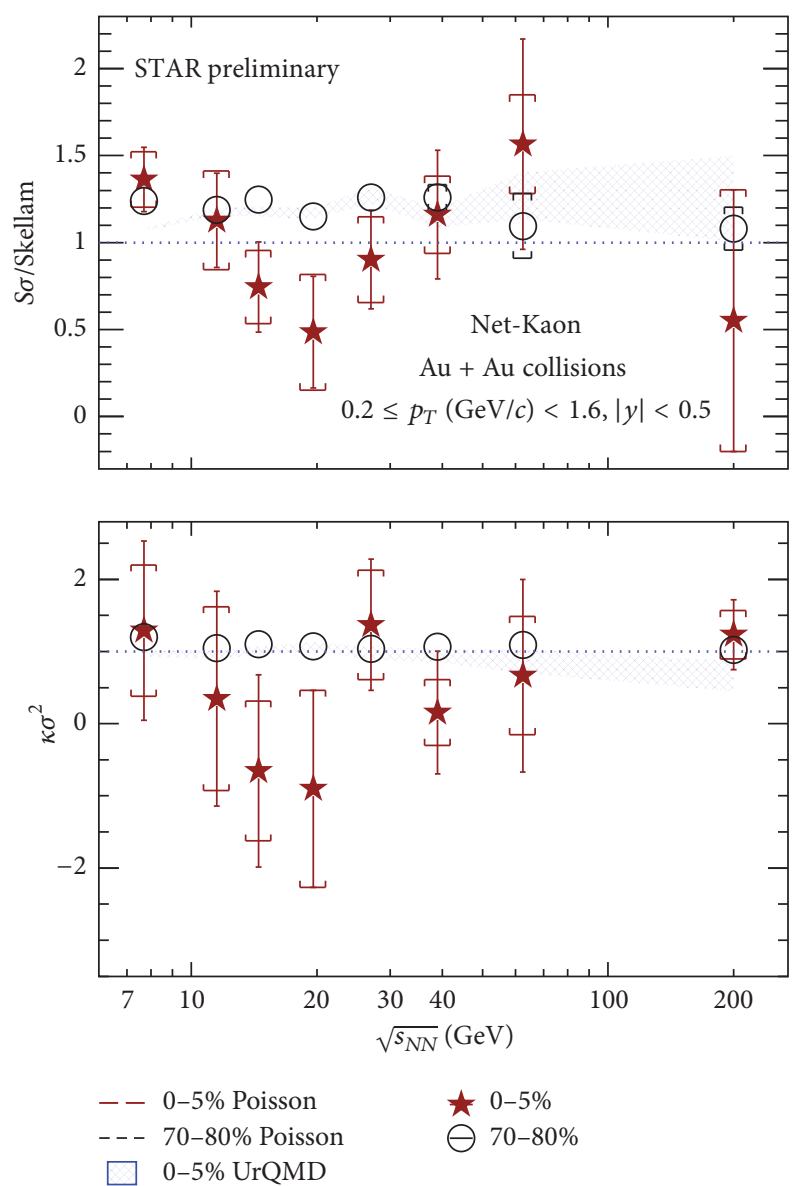

FIGURE 9: Energy dependence of the volume independent cumulant ratios of the net-kaon distributions. Showing $S \sigma /$ Skellam and $\kappa \sigma^{2}$ for top $0-5 \%$ central (red stars) and $70-80 \%$ peripheral (black circles) collisions [67]. The Poisson expectations are denoted as dotted lines and UrQMD calculations are shown as blue bands.

ratios of the lowest-order susceptibilities $\left(M / \sigma^{2}\right)$ for netprotons and net-electric charge. In [38], the freeze-out parameters are extracted using STAR experimental data [64]. $\mu_{B}$ has been extracted using experimentally measured $M / \sigma^{2}$ and lattice calculations, whereas due to the uncertainties on the lattice results in the low-temperature region, it is only possible to extract an upper value for the freeze-out temperature $\left(T_{f} \lesssim 151 \mathrm{MeV}\right)$. In [65], the freeze-out parameters are extracted using $M / \sigma^{2}$ and $S \sigma^{3} / M$ of net-charge from the PHENIX experiment [65] in combination with lattice calculations. Figure 10 shows the direct combination of experimental data and theoretical model calculations to extract physical quantities. The consistency of the results is of fundamental importance to validate that the experimentally created system is close to thermal equilibrium at the freezeout and can be described by lattice QCD simulations, at least in the light quark sector [38].

\section{Summary}

In summary, we have presented a review on the experimental measurements on charge fluctuations over various collision
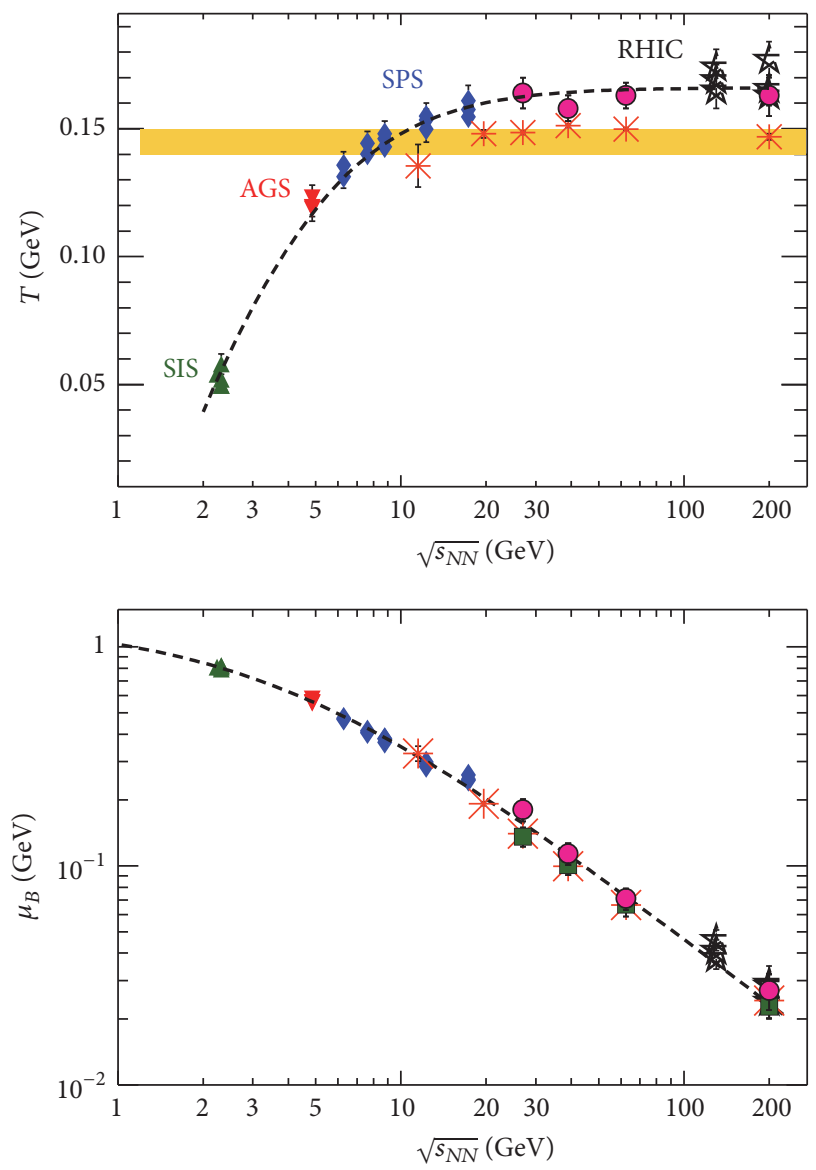

Using cumulants

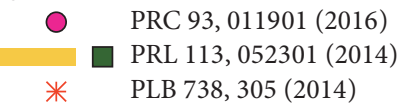

Using particle ratios

$\Delta \nabla \downarrow \hbar--$ Cleymans et al.

Figure 10: The energy dependence of the chemical freeze-out temperature $\left(T_{f}\right)$ and baryon chemical potential $\left(\mu_{B}\right)$ extracted using lattice calculations with experimental measured cumulants $[38,65]$. The freeze-out parameters extracted using HRG and experimental cumulants are also shown [39]. The dashed line is the parameterization given in [77], and the SIS, AGS, SPS, and RHIC data are from [77] and references therein.

energies to search for phase transition and location of the critical point in the QCD phase diagram. We reviewed the results on total charge fluctuations as a function of collision centrality for $\sqrt{s_{N N}}=17.3 \mathrm{GeV}$ in $\mathrm{Pb}+\mathrm{Pb}$ collisions at SPS and for $\sqrt{s_{N N}}=62.4$ and $200 \mathrm{GeV}$ in $\mathrm{Au}+\mathrm{Au}$ collisions at RHIC. It is observed that the relative fluctuations increase from central to peripheral collisions. The fluctuation observables from various models are compared with the experiment data. The results from models show centrality independent behavior. Similar centrality dependence has been observed for total charge fluctuation at the SPS and RHIC experiments. The absence of large dynamical fluctuation in excess of the participant superposition model indicates that there is no 
evidence of critical behavior related to the compressibility observable.

We have also presented the results from net-charge fluctuations from SPS, RHIC, and LHC energies. The netcharge fluctuations in terms of dynamical fluctuation measure $v_{(+-, \text {dyn })}$ as a function of collision centrality and $\sqrt{s_{N N}}$ are studied. $v_{(+-, \text {dyn })}$ increases monotonically from peripheral to central collisions and remains negative indicating the dominance of correlation of positive and negative charged particles. The product of $v_{(+-, \text {dyn })}$ and $\left\langle N_{\mathrm{ch}}\right\rangle$ shows a monotonic decrease with increasing $\sqrt{s_{N N}}$ and approaches to the expectation from a QGP like scenario. For the highest RHIC energy, the measured value of fluctuation is close to the HG prediction, whereas at lower energies the results are higher than HG expectations. At LHC energy $\sqrt{s_{N N}}=2.76 \mathrm{TeV}$, the value of fluctuation observable is significantly lower compared to lower energy results indicating that at LHC energy the fluctuations have their origin in the QGP phase.

The fluctuations of net-charge (net-proton, net-electric charge, and net-kaon) have been measured for various $\sqrt{s_{N N}}$. Deviations are observed for both $S \sigma$ and $\kappa \sigma^{2}$ of net-proton distributions from the Skellam and hadron resonance gas model for $\sqrt{s_{N N}}<39 \mathrm{GeV}$. Maximum deviations from Skellam expectation are observed for $\sqrt{s_{N N}}=19.6$ and $27 \mathrm{GeV}$. The net-electric charge results from both PHENIX and STAR experiments are presented. $S \sigma$ values from both the experiments decrease with increasing $\sqrt{s_{N N}} \cdot \kappa \sigma^{2}$ values from PHENIX remain constant and positive, between $1.0<$ $\kappa \sigma^{2}<2.0$ at all the collision energies within the statistical and systematic uncertainties. However, there is $25 \%$ increase of $\kappa \sigma^{2}$ values at lower energies below $\sqrt{s_{N N}}=39 \mathrm{GeV}$ compared to higher energies. $\kappa \sigma^{2}$ values from STAR experiment are constant at all energies within uncertainties, except for $\sqrt{s_{N N}}=7.7 \mathrm{GeV}$ which shows a negative $\kappa \sigma^{2}$ value. The net-electric charge and net-kaon results do not observe any significant nonmonotonic behavior as a function of collision energy.

To quantify the excess of net-charge fluctuations due to the critical point, different baseline studies have been done by different authors after considering various physical phenomena. On phenomenological side, effects of resonance decay $[78,79]$, proton-antiproton correlations $[71,80]$, kinematic acceptance $[72,75,76]$, nonextensive systems [81], regeneration [39, 82], excluded volume models [83], and so forth are studied. Besides, various studies have been carried out using different Monte Carlo based models, for example, UrQMD [84] and HIJING [85], to compare the experimental results. But it is to be noted that all the above-mentioned physical processes contribute differently at various center of mass energies; therefore, to find a unique ideal baseline estimate to compare the experimental results is still an unsettled issue. The freeze-out parameters are extracted using higher moments of net-charge distributions in combination with lattice QCD and HRG models. The extracted freezeout parameters are in agreement with the thermal model parameterization.

First results from the RHIC BES-I program are intriguing and the statistics collected during BES-I at RHIC are not sufficient to locate the CEP in the QCD phase diagram. Hence, the RHIC BES-II program is proposed to have the precise measurements of these observables to map the QCD phase diagram. The upcoming RHIC BES-II program proposed to be in 2019-20 will cover the $\sqrt{s_{N N}}$ range from 5 to $20 \mathrm{GeV}$, which is the region of interest in the search for a critical point and first-order phase transition, identified by the results from BES-I and by model calculations. In addition, the CBM experiment at FAIR will perform a highprecision study of higher-order fluctuations at various beam energies in order to search for the elusive QCD critical point in the high net-baryon density region: $\mu_{B} \sim 800-500 \mathrm{MeV}$ corresponding to $\sqrt{s_{N N}}=2-4.9 \mathrm{GeV}$ at SIS100. There are other programs at NICA to study the above physics in heavyion collisions in the range $\sqrt{s_{N N}}$ of $4-11 \mathrm{GeV}$. Hence, in the upcoming experimental programs at RHIC BES-II, FAIR, and NICA with upgraded detector system will help to measure the fluctuation variable with reduced uncertainties on the measurements and may find the exact location of the critical point and investigate whole region of phase diagram in more detail.

\section{Disclosure}

The present address of A. K. Mohanty is Saha Institute of Nuclear Physics, 1/AF, Bidhannagar, Kolkata 700064, India.

\section{Competing Interests}

The authors declare that there is no conflict of interests regarding the publication of this manuscript.

\section{References}

[1] M. Stephanov, K. Rajagopal, and E. Shuryak, "Signatures of the tricritical point in QCD," Physical Review Letters, vol. 81, no. 22, pp. 4816-4819, 1998.

[2] M. Alford, K. Rajagopal, and F. Wilczek, "QCD at finite baryon density: nucleon droplets and color superconductivity," Physics Letters, Section B: Nuclear, Elementary Particle and High-Energy Physics, vol. 422, no. 1-4, pp. 247-256, 1998.

[3] M. A. Stephanov, "Random matrix model of QCD at finite density and the nature of the quenched limit," Physical Review Letters, vol. 76, no. 24, pp. 4472-4475, 1996.

[4] Y. Aoki, G. Endrodi, Z. Fodor, S. D. Katz, and K. K. Szabó, “The order of the quantum chromodynamics transition predicted by the standard model of particle physics," Nature, vol. 443, no. 7112, pp. 675-678, 2006.

[5] K. Fukushima and T. Hatsuda, "The phase diagram of dense QCD," Reports on Progress in Physics, vol. 74, no. 1, Article ID 014001, 2011.

[6] M. Stephanov, "QCD phase diagram and the critical point," Progress of Theoretical Physics Supplement, vol. 153, pp. 139-156, 2004.

[7] Z. Fodor and S. D. Katz, "Critical point of QCD at finite T and $\mu$, lattice results for physical quark masses," Journal of High Energy Physics, vol. 4, article 50, 2004.

[8] M. Stephanov, K. Rajagopal, and E. Shuryak, "Event-by-event fluctuations in heavy ion collisions and the QCD critical point," Physical Review D, vol. 60, no. 11, Article ID 114028, 1999. 
[9] S. Mukherjee, R. Venugopalan, and Y. Yin, "Real-time evolution of non-Gaussian cumulants in the QCD critical regime," Physical Review C, vol. 92, no. 3, Article ID 034912, 2015.

[10] S. Mukherjee, R. Venugopalan, and Y. Yin, "Universal offequilibrium scaling of critical cumulants in the QCD phase diagram," Physical Review Letters, vol. 117, Article ID 222301, 2016.

[11] STAR Collaboration, "Studying the phase diagram of QCD matter at RHIC," STAR Notes SN0598, 2014, https://drupal.star.bnl .gov/STAR/starnotes/public/sn0598.

[12] R. Rapp, B. Kämpfer, A. Andronic et al., "In-medium excitations," in The CBM Physics Book: Compressed Baryonic Matter in Laboratory Experiments, vol. 814 of Lecture Notes in Physics, pp. 335-529, Springer, Berlin, Germany, 2011.

[13] Design and construction of nuclotron-based ion collider facility (NICA) conceptual design report, 2008, http://nica.jinr.ru/files/ NICA_CDR.pdf.

[14] “J-PARC Heavy Ion Project," http://asrc.jaea.go.jp/soshiki/gr/ hadron/jparc-hi/documents.html.

[15] M. Asakawa, U. Heinz, and B. Müller, "Fluctuation probes of quark deconfinement," Physical Review Letters, vol. 85, no. 10, article no. 2072, 2000.

[16] L. Stodolsky, "Temperature fluctuations in multiparticle production," Physical Review Letters, vol. 75, no. 6, pp. 1044-1045, 1995.

[17] J. Adams and STAR Collaboration, "Net charge fluctuations in $\mathrm{Au}+\mathrm{Au}$ collisions at $\sqrt{s_{N N}}=130 \mathrm{GeV}$," Physical Review C, vol. 68, no. 4, Article ID 044905, 2003.

[18] B. I. Abelev, M. M. Aggarwal, Z. Ahammed et al., " $K / \pi$ fluctuations at relativistic energies," Physical Review Letters, vol. 103, Article ID 092301, 2009.

[19] C. Alt and NA49 Collaboration, "Energy dependence of particle ratio fluctuations in central $\mathrm{Pb}+\mathrm{Pb}$ collisions from $\sqrt{s_{N N}}=6.3$ to 17.3 GeV," Physical Review C, vol. 79, no. 4, Article ID 044910, 2009.

[20] K. Adcox and PHENIX Collaboration, "Event-by-event fluctuations in mean $p_{T}$ and mean $e_{T}$ in $\sqrt{s_{N N}}=130 \mathrm{GeV} \mathrm{Au}+\mathrm{Au}$ collisions," Physical Review C, vol. 66, no. 2, Article ID 024901, 2002.

[21] S. S. Adler and PHENIX Collaboration, "Measurement of nonrandom event-by-event fluctuations of average transverse momentum in $\sqrt{s_{N N}}=200 \mathrm{GeV} \mathrm{Au}+\mathrm{Au}$ and $\mathrm{p}+\mathrm{p}$ collisions," Physical Review Letters, vol. 93, no. 9, Article ID 092301, 2004.

[22] S. Jeon and V. Koch, "Charged particle ratio fluctuation as a signal for Quark-Gluon plasma," Physical Review Letters, vol. 85, no. 10 , article 2076, 2000.

[23] S. Mrówczyński, "Hadronic matter compressibility from eventby-event analysis of heavy-ion collisions," Physics Letters B, vol. 430, no. 1-2, pp. 9-14, 1998.

[24] V. V. Begun, M. I. Gorenstein, A. P. Kostyuk, and O. S. Zozulya, "Particle number fluctuations in the microcanonical ensemble," Physical Review C, vol. 71, no. 5, 2005.

[25] S. Jeon and V. Koch, "Event-by-event fluctuations," in QuarkGluon Plasma, R. C. Hwa and X.-N. Wang, Eds., pp. 430-490, World Scientific, Singapore, 2003.

[26] V. V. Begun, M. Gaździcki, M. I. Gorenstein, and O. S. Zozulya, "Particle number fluctuations in a canonical ensemble," Physical Review C, vol. 70, no. 3, Article ID 034901, 2004.

[27] F. Becattini, A. Keränen, L. Ferroni, and T. Gabbriellini, "Multiplicity fluctuations in a hadron gas with exact conservation laws," Physical Review C, vol. 72, no. 6, 2005.
[28] P. Garg, D. K. Mishra, P. K. Netrakanti, and A. K. Mohanty, "Multiplicity fluctuations in heavy-ion collisions using canonical and grand-canonical ensemble," European Physical Journal A, vol. 52, no. 2, 2016.

[29] H. Stanley, Introduction to Phase Transitions and Critical Phenomena, Oxford University Press, Oxford, UK, 1971.

[30] A. Adare and PHENIX Collaboration, "Charged hadron multiplicity fluctuations in $\mathrm{Au}+\mathrm{Au}$ and $\mathrm{Cu}+\mathrm{Cu}$ collisions from $\sqrt{s_{N N}}=22.5$," Physical Review C, vol. 78, no. 4, Article ID $044902,2008$.

[31] B. -J. Schaefer and J. Wambach, "Susceptibilities near the QCD (tri)critical point," Physical Review D, vol. 75, no. 8, Article ID 085015, 2007.

[32] C. Sasaki, B. Friman, and K. Redlich, "Quark number fluctuations in a chiral model at finite baryon chemical potential," Physical Review D, vol. 75, no. 5, Article ID 054026, 2007.

[33] V. Koch, A. Majumder, and J. Randrup, "Baryon-strangeness correlations: a diagnostic of strongly interacting matter," Physical Review Letters, vol. 95, no. 18, 2005.

[34] M. A. Stephanov, "Sign of kurtosis near the QCD critical point," Physical Review Letters, vol. 107, no. 5, Article ID 052301, 2011.

[35] M. Asakawa, S. Ejiri, and M. Kitazawa, "Third moments of conserved charges as probes of QCD phase structure," Physical Review Letters, vol. 103, no. 26, 2009.

[36] S. Ejiri, F. Karsch, and K. Redlich, "Hadronic fluctuations at the QCD phase transition," Physics Letters, Section B: Nuclear, Elementary Particle and High-Energy Physics, vol. 633, no. 2-3, pp. 275-282, 2006.

[37] A. Bazavov, H.-T. Ding, P. Hegde et al., "Freeze-out conditions in heavy ion collisions from QCD thermodynamics," Physical Review Letters, vol. 109, no. 19, Article ID 192302, 2012.

[38] S. Borsanyi, Z. Fodor, S. D. Katz, S. Krieg, C. Ratti, and K. K. Szabo, "Freeze-out parameters from electric charge and baryon number fluctuations: is there consistency?" Physical Review Letters, vol. 113, no. 5, Article ID 052301, 2014.

[39] P. Alba, W. Alberico, R. Bellwied et al., "Freeze-out conditions from net-proton and net-charge fluctuations at RHIC," Physics Letters B, vol. 738, pp. 305-310, 2014.

[40] M. M. Aggarwal, A. Agnihotri, Z. Ahammed et al., "Localized charged-neutral fluctuations in $158 \mathrm{~A} \mathrm{GeV} \mathrm{Pb}+\mathrm{Pb}$ collisions," Physical Review C, vol. 64, Article ID 011901, 2001.

[41] K. Rajagopal and F. Wilczek, "Emergence of coherent long wavelength oscillations after a quench: application to QCD," Nuclear Physics, Section B, vol. 404, no. 3, pp. 577-589, 1993.

[42] H. Heiselberg, "Event-by-event physics in relativistic heavy-ion collisions," Physics Reports, vol. 351, no. 3, pp. 161-194, 2001.

[43] C. Alt, T. Antičić, B. Baatar et al., "Centrality and system size dependence of multiplicity fluctuations in nuclear collisions at 158A GeV," Physical Review C, vol. 75, no. 6, Article ID 064904, 2007.

[44] V. P. Konchakovski, S. Haussler, M. I. Gorenstein, E. L. Bratkovskaya, M. Bleicher, and H. Stöcker, "Particle number fluctuations in high-energy nucleus-nucleus collisions from microscopic transport approaches," Physical Review C: Nuclear Physics, vol. 73, no. 3, Article ID 034902, 2006.

[45] G. Baym and H. Heiselberg, "Event-by-event fluctuations in ultrarelativistic heavy-ion collisions," Physics Letters, Section B: Nuclear, Elementary Particle and High-Energy Physics, vol. 469, no. 1-4, pp. 7-11, 1999.

[46] M. Gyulassy and X.-N. Wang, "HIJING 1.0: a Monte Carlo program for parton and particle production in high energy 
hadronic and nuclear collisions," Computer Physics Communications, vol. 83, no. 2-3, pp. 307-331, 1994.

[47] W. Cassing and E. L. Bratkovskaya, "Hadronic and electromagnetic probes of hot and dense nuclear matter," Physics Report, vol. 308, no. 2-3, pp. 65-233, 1999.

[48] M. Bleicher, E. Zabrodin, C. Spieles et al., "Relativistic hadronhadron collisions in the ultra-relativistic quantum molecular dynamics model," Journal of Physics G, vol. 25, pp. 1859-1896, 1999.

[49] K. Werner, "Strings, pomerons and the VENUS model of hadronic interactions at ultrarelativistic energies," Physics Reports, vol. 232, no. 2-5, pp. 87-299, 1993.

[50] C. Pruneau, S. Gavin, and S. Voloshin, "Methods for the study of particle production fluctuations," Physical Review C, vol. 66, no. 4, Article ID 044904, 2002.

[51] B. I. Abelev and STAR Collaboration, "Beam-energy and system-size dependence of dynamical net charge fluctuations," Physical Review C, vol. 79, no. 2, Article ID 024906, 2009.

[52] H. Sako and H. Appelshäuser, "Event-by-event fluctuations at 40, 80 and $158 \mathrm{~A} \mathrm{GeV/c} \mathrm{in} \mathrm{Pb}+$ Au collisions," Journal of Physics G: Nuclear and Particle Physics, vol. 30, no. 8, p. S1371, 2004.

[53] B. Abelev and ALICE Collaboration, "Net-charge fluctuations in $\mathrm{Pb}-\mathrm{Pb}$ collisions at $\sqrt{s_{N N}}=2.76 \mathrm{TeV}$," Physical Review Letters, vol. 110, no. 15, Article ID 152301, 2013.

[54] E. V. Shuryak and M. A. Stephanov, "Long-range charge fluctuations and search for a quark-gluon plasma signal," Physical Review C: Nuclear Physics, vol. 63, no. 6, Article ID 064903, 2001.

[55] M. A. Aziz and S. Gavin, "Causal diffusion and the survival of charge fluctuations in nuclear collisions," Physical Review C, vol. 70, no. 3, Article ID 034905, 2004.

[56] S. Gavin, "Traces of thermalization from $p t$ fluctuations in nuclear collisions," Physical Review Letters, vol. 92, no. 16, Article ID 162301, 2004.

[57] F. W. Bopp and J. Ranft, "Charged particle fluctuation as signal of the dynamics in heavy ion processes," The European Physical Journal C, vol. 22, no. 1, pp. 171-177, 2001.

[58] R. Singh, L. Kumar, P. K. Netrakanti, and B. Mohanty, "Selected experimental results from heavy-ion collisions at LHC," Advances in High Energy Physics, vol. 2013, Article ID 761474, 22 pages, 2013.

[59] B. Friman, C. Höhne, J. Knoll et al., Eds., The CBM Physics Book: Compressed Baryonic Matter in Laboratory Experiments, vol. 814 of Lecture Notes in Physics, Springer, 2011.

[60] M. A. Stephanov, "Non-Gaussian fluctuations near the QCD critical point," Physical Review Letters, vol. 102, no. 3, Article ID 032301, 2009.

[61] Y. Hatta and M. A. Stephanov, "Proton-number fluctuation as a signal of the QCD critical end point," Physical Review Letters, vol. 91, no. 10, Article ID 102003, 2003, Erratum to: Physical Review Letters, vol. 91, Article ID 129901, 2003.

[62] M. M. Aggarwal, Z. Ahammed, A. V. Alakhverdyants et al., "Higher moments of net proton multiplicity distributions at RHIC," Physical Review Letters, vol. 105, no. 2, Article ID 022302, 2010.

[63] L. Adamczyk, J. K. Adkins, G. Agakishiev et al., "Energy dependence of moments of net-proton multiplicity distributions at RHIC," Physical Review Letters, vol. 112, no. 3, Article ID 032302, 7 pages, 2014.

[64] L. Adamczyk, J. K. Adkins, G. Agakishiev et al., "Beam energy dependence of moments of the net-charge multiplicity distributions in $\mathrm{Au}+\mathrm{Au}$ collisions at RHIC," Physical Review Letters, vol. 113, Article ID 092301, 2014.
[65] A. Adare, S. Afanasiev, C. Aidala et al., "Measurement of higher cumulants of net-charge multiplicity distributions in $\mathrm{Au}+\mathrm{Au}$ collisions at $\sqrt{s_{N N}}=7.7-200 \mathrm{GeV}$," Physical Review C, vol. 93, no. 1, Article ID 011901, 2016.

[66] A. Sarkar, "Higher moments of net kaon multiplicity distributions at RHIC energies for the search of QCD Critical Point at STAR," Journal of Physics: Conference Series, vol. 509, Article ID 012069, 2014.

[67] J. Thäder, "Higher moments of net-particle multiplicity distributions," Nuclear Physics A, vol. 956, pp. 320-323, 2016.

[68] F. Karsch and K. Redlich, "Probing freeze-out conditions in heavy ion collisions with moments of charge fluctuations," Physics Letters B, vol. 695, no. 1-4, pp. 136-142, 2011.

[69] S. Gupta, X. Luo, B. Mohanty, H. G. Ritter, and N. Xu, "Scale for the phase diagram of quantum chromodynamics," Science, vol. 332, no. 6037, pp. 1525-1528, 2011.

[70] X. Luo, "Error estimation for moment analysis in heavy-ion collision experiment," Journal of Physics G: Nuclear and Particle Physics, vol. 39, no. 2, Article ID 025008, 2012.

[71] D. K. Mishra, P. Garg, and P. K. Netrakanti, "Effect of correlations on cumulants in heavy-ion collisions," Physical Review C: Nuclear Physics, vol. 93, no. 2, Article ID 024918, 2016.

[72] P. Garg, D. K. Mishra, P. K. Netrakanti et al., "Conserved number fluctuations in a hadron resonance gas model," Physics Letters, Section B: Nuclear, Elementary Particle and High-Energy Physics, vol. 726, no. 4-5, pp. 691-696, 2013.

[73] V. Skokov, B. Friman, and K. Redlich, "Non-perturbative dynamics and charge fluctuations in effective chiral models," Physics Letters B, vol. 708, no. 1-2, pp. 179-185, 2012.

[74] M. Kitazawa and M. Asakawa, "Revealing baryon number fluctuations from proton number fluctuations in relativistic heavy ion collisions," Physical Review C, vol. 85, no. 2, 2012.

[75] F. Karsch, K. Morita, and K. Redlich, "Effects of kinematic cuts on net electric charge fluctuations," Physical Review C, vol. 93, no. 3, Article ID 034907, 2016.

[76] B. Ling and M. A. Stephanov, "Acceptance dependence of fluctuation measures near the QCD critical point," Physical Review C, vol. 93, no. 3, Article ID 034915, 2016.

[77] J. Cleymans, H. Oeschler, K. Redlich, and S. Wheaton, "Comparison of chemical freeze-out criteria in heavy-ion collisions," Physical Review C: Nuclear Physics, vol. 73, no. 3, Article ID 034905, 2006.

[78] D. K. Mishra, P. Garg, P. K. Netrakanti, and A. K. Mohanty, "Effect of resonance decay on conserved number fluctuations in a hadron resonance gas model," Physical Review C, vol. 94, no. 1,2016 .

[79] M. Nahrgang, M. Bluhm, P. Alba, R. Bellwied, and C. Ratti, "Impact of resonance regeneration and decay on the net proton fluctuations in a hadron resonance gas," The European Physical Journal C, vol. 75, no. 12, article 573, 2015.

[80] A. Bzdak, V. Koch, and N. Strodthoff, "Cumulants and correlation functions vs the QCD phase diagram," https://arxiv.org/ abs/1607.07375.

[81] D. K. Mishra, P. Garg, P. K. Netrakanti, and A. K. Mohanty, "Netbaryon number fluctuations with the hadron resonance gas model using Tsallis distribution," Journal of Physics G: Nuclear and Particle Physics, vol. 42, no. 10, Article ID 105105, 2015.

[82] M. Kitazawa and M. Asakawa, "Relation between baryon number fluctuations and experimentally observed proton number fluctuations in relativistic heavy ion collisions," Physical Review C, vol. 86, no. 2, Article ID 024904, 2012, Erratum to: Physical Review C, vol. 86, Article ID 069902, 2012. 
[83] J. Fu, "Higher moments of net-proton multiplicity distributions in heavy ion collisions at chemical freeze-out," Physics Letters B, vol. 722, no. 1-3, pp. 144-150, 2013.

[84] G. D. Westfall, "UrQMD study of the effects of centrality definitions on higher moments of net protons at energies available at the BNL Relativistic Heavy Ion Collider," Physical Review C, vol. 92, no. 2, Article ID 024902, 7 pages, 2015.

[85] T. J. Tarnowsky and G. D. Westfall, "First study of the negative binomial distribution applied to higher moments of net-charge and net-proton multiplicity distributions," Physics Letters B, vol. 724, no. 1-3, pp. 51-55, 2013. 

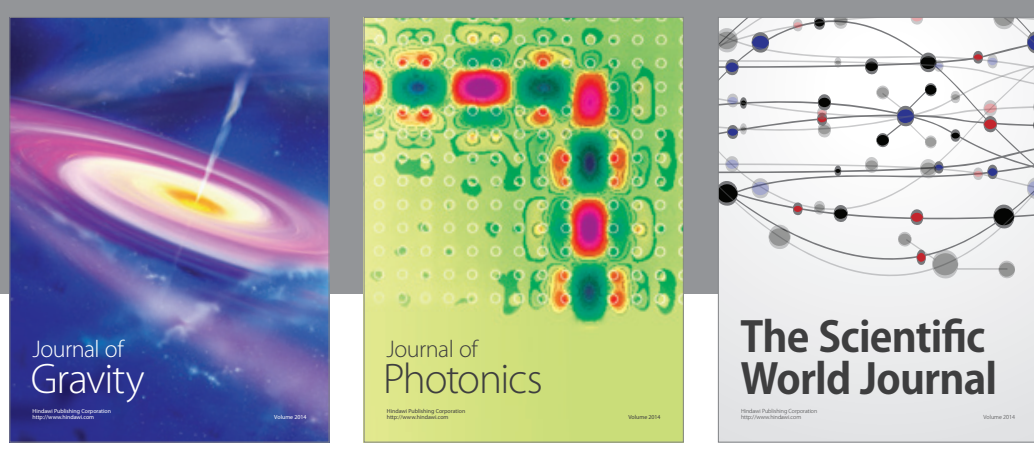

The Scientific World Journal
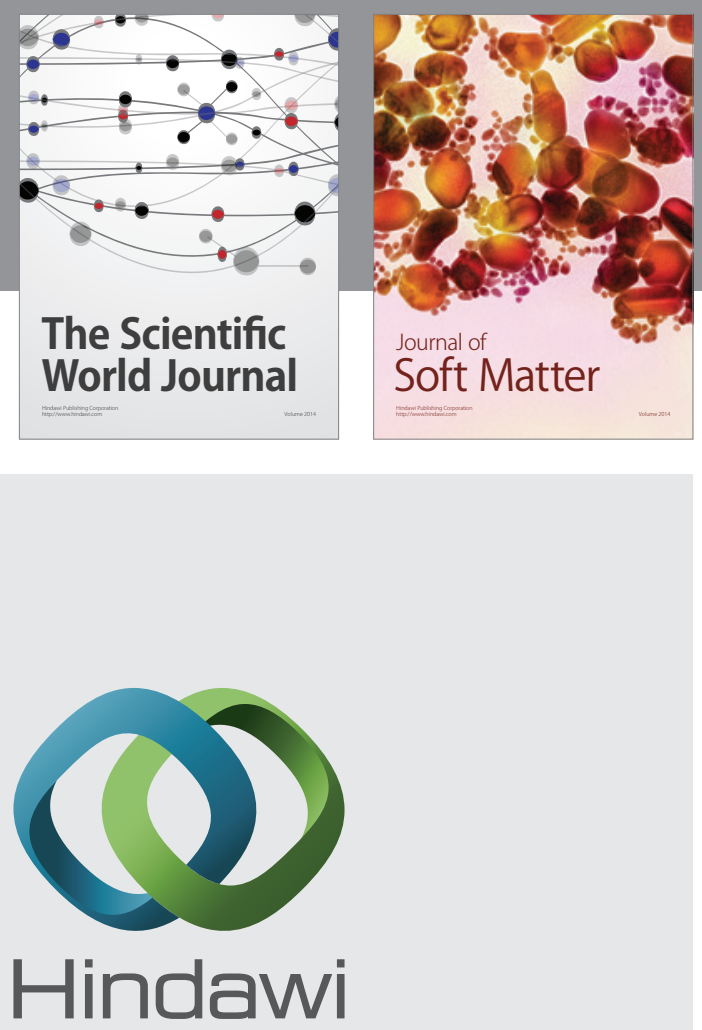

Submit your manuscripts at

https://www.hindawi.com
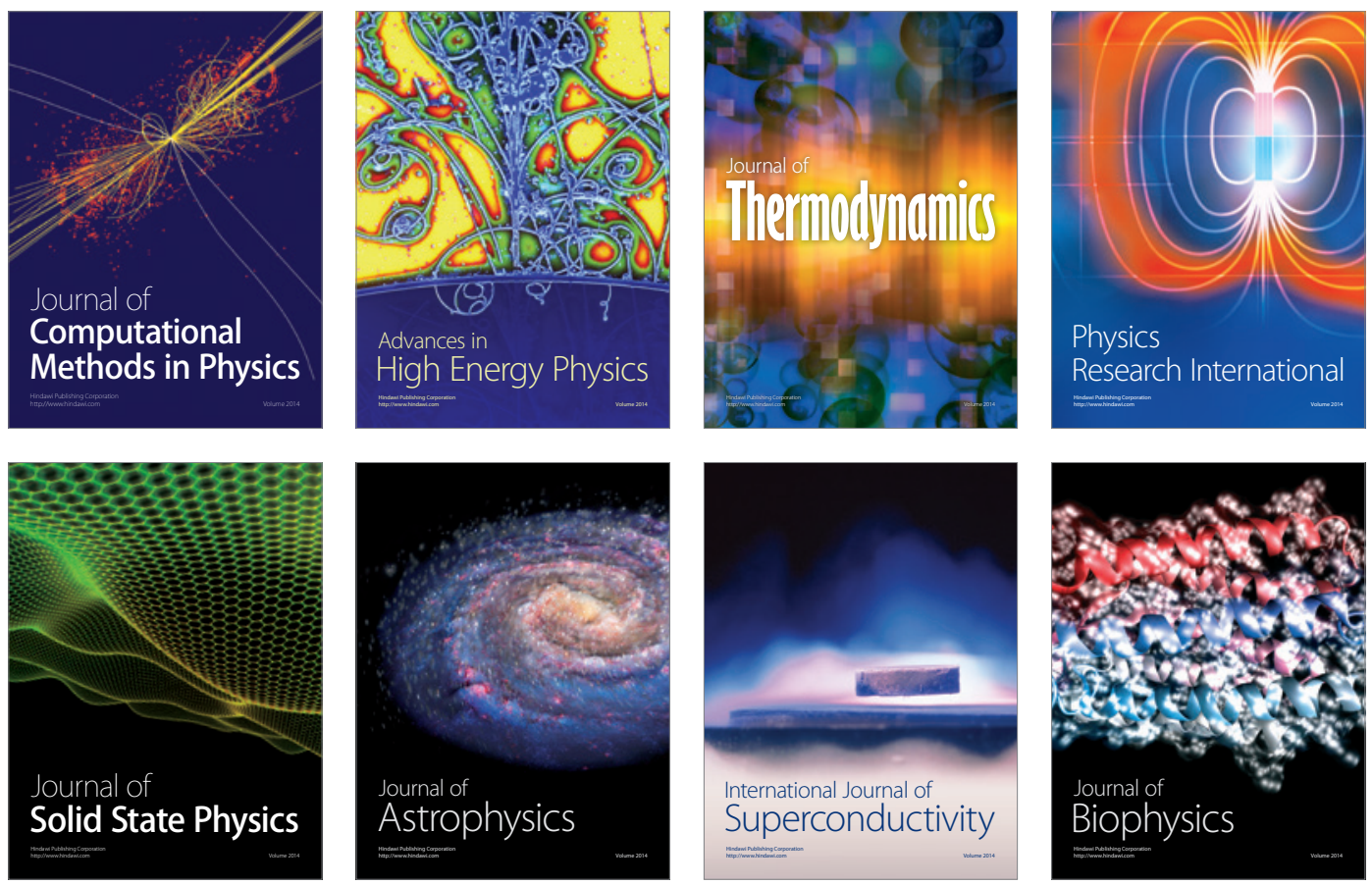
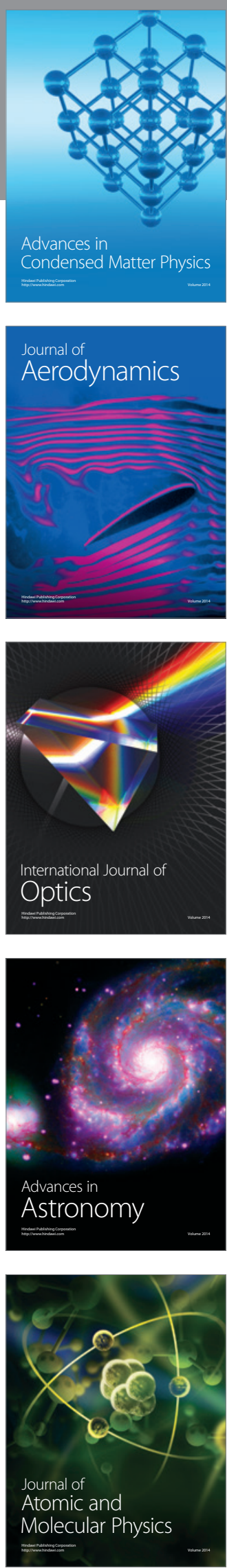